\title{
Activités
}

18-1 | 2021

Le programme de recherche cours d'action (2)

\section{Analyse du cours de l'action et projet anthropocentré : contributions à la conception de systèmes automatisés}

Analysis of the Course of Action and Anthropocentric Project: contributions to the design of automated systems

Francisco de Paula Antunes Lima, Rodrigo Ribeiro, Marcelle La Guardia et Samira Nagem

\section{OpenEdition \\ Journals}

Édition électronique

URL : http://journals.openedition.org/activites/6098

DOI : 10.4000/activites.6098

ISSN : $1765-2723$

Éditeur

ARPACT - Association Recherches et Pratiques sur les ACTivités

\section{Référence électronique}

Francisco de Paula Antunes Lima, Rodrigo Ribeiro, Marcelle La Guardia et Samira Nagem, «Analyse du cours de l'action et projet anthropocentré : contributions à la conception de systèmes automatisés », Activités [En ligne], 18-1 | 2021, mis en ligne le 15 avril 2021, consulté le 17 avril 2021. URL : http://journals.openedition.org/activites/6098 ; DOI : https://doi.org/10.4000/activites.6098

Ce document a été généré automatiquement le 17 avril 2021

\section{(c) (†) $\ominus$}

Activités est mis à disposition selon les termes de la licence Creative Commons Attribution - Pas d'Utilisation Commerciale - Pas de Modification 4.0 International 


\title{
Analyse du cours de l'action et projet anthropocentré : contributions à la conception de systèmes automatisés
}

\author{
Analysis of the Course of Action and Anthropocentric Project: contributions to \\ the design of automated systems
}

Francisco de Paula Antunes Lima, Rodrigo Ribeiro, Marcelle La Guardia et Samira Nagem

\section{NOTE DE L'ÉDITEUR}

Article soumis le 11/03/2020, accepté le 11/09/2020

Nous remercions le CEI qui a permis la réalisation et la diffusion des résultats de la recherche de développement technologique, effectuée par l'équipe de Situated Consultoria e Pesquisa.

\section{Introduction}

1 Dans le champ de l'ergonomie, le Programme de Recherche du Cours de l'Action (PRCA) se démarque par trois aspects: (i) globalité des pôles scientifiques, méthodologiques, philosophiques et technologiques pris en compte dans le programme de forme organique; (ii) formalisation de présupposés ontologiques (sociaux et existentiels), épistémologiques, technologiques et méthodologiques (description empirique, analyse multi-niveaux) en mettant l'accent sur la théorie de l'activité; (iii) analyse de la relation entre l'activité humaine et l'activité politique; (iv) proposition d'intégration entre les dimensions micro et macrosociales par des analyses multi-niveaux afin d'intégrer économie et activité humaine et, du point de vue pratique, élargir 
l'ingénierie des situations à l'espace et à l'organisation; (v) proposition d'une théorie de la subjectivité ou une philosophie de l'expérience (un monisme avec de la subjectivité) cohérente avec l'activité et la cognition situées sans avoir besoin de recourir à un concept de sujet qui déboucherait sur une conception duelle de la relation sujet-objet.

2 Le PPCA est présent dans divers ouvrages (Pinsky, 1992; Theureau, 1992; Theureau, 2004 ; Theureau, 2006; Theureau, 2009; Theureau, 2015 ; Theureau, 2019; Theureau \& Jeffroy, 1994) dont la complexité rend le contexte et ses aspects spécifiques difficiles à appréhender pour les chercheurs qui assument des perspectives similaires, même pour les ergonomistes. Ces difficultés vont de la recherche empirique, considérée très " lourde », aux réflexions théoriques plus avancées.

3 Cet article aborde un aspect spécifique du Programme de Recherche Technologique (PRT), un pôle interne du PRCA. La question étudiée est la contribution du PRCA à la conception ergonomique en démontrant son efficacité dans des situations complexes, même sans avoir recours au système conceptuel dans son ensemble et sans développer de modèles détaillés du cours de l'action. Actuellement, dans le champ même de l'ergonomie de l'activité, on peut observer un retour en arrière théorique et méthodologique de l'analyse de l'activité. Cette affirmation peut sembler paradoxale, mais le mouvement de développement de cadres théoriques pour étayer les interventions ergonomiques, qui a débuté dans les années 1980 et au sein duquel la théorie du cours de l'action occupa une place centrale (Wisner, 2004), s'est engagé sur une voie au sein de laquelle l'ergonomie se retrouve sans base théorique nécessaire à la description empirique de son objet central : l'activité de travail. Sans cet élément, comme nous en discuterons par la suite, l'intervention ergonomique est limitée en capacité pour orienter les projets d'ingénierie afin de les rendre plus compatibles avec l'activité du travail.

4 Les autres tendances élaborées dans les années 1980 n'ont pas priorisé l'élaboration d'une théorie de l'activité de travail. En termes de formalisation plus générale de la méthode, dans Comprendre le travail pour le transformer, l'activité est située entre le travail prescrit et le travail réel, elle est couramment reproduite dans la différence entre la tâche et l'activité sans que cette " activité » soit placée au centre des réflexions théoriques à partir de descriptions empiriques pertinentes. De manière symptomatique, la tâche a été dédoublée en tâche réelle et tâche effective alors que l'activité est toujours encore un concept non analysé tout comme "l'expérience ».

5 Même lorsque l'ergologie s'est appropriée l'ergonomie en tant que propédeutique et bien que l'activité de travail, et ensuite l'activité humaine, soit placée comme objet central de la réflexion, elle a plutôt servi à fonder une philosophie de l'activité qu'une praxéologie fondée empiriquement. Il ne s'agit pas de mettre en question le potentiel théorique de l'ergologie et sa capacité de transformer le travail, mais de son efficacité limitée d'action au sein de projets d'ingénierie.

De nos jours, les ergonomistes se rapprochent de la théorie d'Engeström qui a un certain intérêt au niveau de la conduite de l'intervention. Cependant, ses modèles analytiques, décrits en termes de système d'activités, sont encore trop statiques pour décrire empiriquement la dynamique de l'activité dans toutes ses nuances et complexités, plus spécialement ses moments de créativité. La notion « d'apprentissage expansif » (Engeström, 1987) laisse penser qu'il existe une analyse de la dynamique de l'activité, mais la description empirique se résume à constater les états consécutifs sans 
que les processus actifs et cognitifs dynamiques sous-jacents à l'expansion ne soient mis en évidence et analysés.

7 Même dans la clinique de l'activité, étayée par des concepts dynamiques et priorisant la transformation par rapport à la compréhension avec son axe privilégié du « réel de l'activité »(Clot, 2010), la description de l'activité réelle est, par définition, encore limitée et l'accent est mis sur les controverses professionnelles et le jeu complexe de destination subjective des actions à divers agents, auxquels il est attribué des résultats observables en termes de développement de l'activité et du métier (voir, par exemple, Fernandez, 2004 et Simonet, 2009). Il ne s'agit pas ici de questionner les options théoriques de la clinique de l'activité ou sa capacité de produire des transformations du travail, mais la pertinence de la priorité de la transformation par rapport à la compréhension lorsqu'il s'agit d'intervenir dans de grands projets technologiques, même si le rôle central des instruments est reconnu par les références à la théorie de l'activité instrumentale de Vygotski.

8 Les « espaces de débat sur le travail » (EDT), qui ont tendance aujourd'hui à se diluer dans les interventions ergonomiques (Falzon, 2013; Rocha, 2015; Rocha, Mollo, \& Daniellou, 2015), sont également économes en termes d'analyses systématiques et approfondies de l'activité de travail et privilégient les dispositifs d'intervention qui comprennent, de façon directe, des méthodologies d'analyse menées par les travailleurs eux-mêmes. Rocha (2015), par exemple, anime les EDT sur la base d'évidences enregistrées par des photographies prises par des électriciens, ce qui restreint évidemment les analyses à ce qui est directement noté et photographié et rapproche l'analyse ergonomique aux espaces d'expression, comme proposé dans l'Analyse Collective du Travail (Análise Coletiva do Trabalho) développée par Leda Leal Ferreira (2015), réalisée sans accès direct aux situations de travail. Les EDT se rapprochent également des Groupes de Rencontre du Travail (GET), utilisés en ergologie en tant que méthode d'expression de l'expérience (Trinquet, 2010). Pourtant, si l'ergologie, dont le point fort est d'être une philosophie du travail, peut se satisfaire de groupes d'expression, dans le cas de l'ergonomie, il s'agit d'un net pas en arrière, voire un contresens, lorsque l'on tient compte de son objectif de transformer le travail par le projet de nouvelles situations.

Dans l'ensemble, ces approches plus récentes, soit de l'ergonomie ou des cliniques du travail, finissent par limiter la contribution des analystes du travail quant aux solutions de problèmes plus complexes exigeant des analyses qui puissent appréhender la dynamique de l'activité réelle dans des situations concrètes. À titre d'exemple, nous pouvons citer les problèmes qui intègrent plusieurs niveaux organisationnels, de l'activité individuelle-collective aux interfaces organisationnelles, ou des projets technologiques complexes, comme les systèmes automatisés et les salles de contrôle (Lima, Duarte, Resende, Garrigou, \& Carballeda, 2015).

10 Toutes ces approches peuvent contribuer aux changements et transformations positives des conditions de travail et au développement de l'activité elle-même, mais ceci est réalisé sans formalisation rigoureuse de l'activité réelle et des méthodes nécessaires d'analyse de l'activité, assise de son développement ultérieur. Sans nul doute que des projets menés selon la démarche de l'ergonomie de conception, que ce soit par dans la conduite de projets (Daniellou, 2007) ou dans l'élaboration d'artefacts (Folcher \& Rabardel, 2007), permettent de créer des instruments plus appropriés aux travailleurs et usagers ${ }^{1}$. Cependant, même dans ces cas, les aspects plus complexes et 
dynamiques sont encore laissés aux mains des concepteurs ou à l'expression spontanée des travailleurs participant aux projets collaboratifs. Les cas empiriques discutés dans cet article ne démontrent pas uniquement la pertinence de la théorie du cours de l'action au sein des projets de nouvelles situations de travail, mais également que la praxéologie empirique décrivant la dynamique de l'activité de l'activité est nécessaire pour traiter des interfaces $\mathrm{H}-\mathrm{H}$ (formation et organisation du travail) et $\mathrm{H}-\mathrm{M}$ (automation) si nous voulons faire face à des problèmes plus complexes avec des propriétés dynamiques internes de systèmes automatisés (H-H-M).

11 Les problèmes de projet d'interfaces ne sont pas nouveaux. Depuis la diffusion de l'automation dans les années 1950, des tentatives de développement de technologies anthropocentriques se sont succédé sans grand succès. Les analyses proposées dans cet article permettent de formuler l'hypothèse que l'une des principales raisons de cet échec historique était la non-existence d'une théorie de l'activité avec des ressources conceptuelles et méthodologiques permettant effectivement de projeter les interfaces entre les hommes et les machines ou, en d'autres termes, d'intégrer les aspects social et technique au sein de projets sociotechniques. L'histoire récente démontre que les principes généraux des approches anthropocentriques sont importants pour ouvrir la voie à des projets sociotechniques, mais encore peu opérationnels pour intégrer les aspects sociaux, subjectifs et techniques.

12 Les cas empiriques analysés dans cet article proviennent d'une intervention dans une usine de génération d'électricité qui opère de Petites Centrales Hydro-électriques ( $\mathrm{PCHs})$. Au moment de l'intervention, l'usine passait par une réorganisation importante avec l'automation des PCHs et l'opération en salle de contrôle à distance (à $100 \mathrm{~km}$ de distance), ce qui impliquait une réduction du personnel opérationnel et une organisation du travail en équipes. Les travailleurs, affectés auparavant dans des PCHs spécifiques devaient devenir polyvalents. Pour ce motif, il existait aussi une demande de formalisation des savoirs tacites des opérateurs de terrain qui allaient partir à la retraite, quitter l'entreprise ou contrôler plus d'une $\mathrm{PCH}$.

13 Nous évoquerons les produits développés durant cette intervention qui peuvent aussi être utilisés dans d'autres situations de travail et/ou projets: (1) «arbres de jugements » et « arbres séquentiels » basés sur le cours de l'action des opérateurs pour opérationnaliser le concept «d'objets intermédiaires de la formation » (analogues aux " objets intermédiaires de conception " proposés par Vinck, 2013); (2) un «modèle analytique d'automation anthropocentrique et appropriable», sur la base de representamens caractéristiques des activités critiques analysées; et (3) un «modèle analytique de co-opération: opération locale versus opération à distance » qui agit comme un « objet intermédiaire de co-opération » entre les opérateurs de terrain et la salle de contrôle à distance.

Outre la contribution technologique au PRT, les produits résultants de cette intervention tentent également de résoudre une question encore non étudiée jusqu'alors dans le PRCA: comment agir sur ce que l'on ne peut expliquer dans l'activité, c'est-à-dire sur la partie tacite de l'expérience qui ne peut être relatée, montrée ou commentée ? On sait que les descriptions du cours de l'action servent à projeter des situations de travail diverses. Cependant, hormis cet usage direct, les arbres de "jugements" et "séquentiels " permettent que les jugements tacites effectués par les opérateurs expérimentés soient partiellement explicités et discutés entre spécialistes eux-mêmes (créant un partage et un débat sur les différents 
«styles ») et en interaction avec les novices (en attirant l'attention de ces derniers) ${ }^{2}$. L'organisation des jugements tacites explicités dans les arbres, du plus simple au plus complexe ou dans le cadre de la séquence d'actions d'une activité, fonctionne comme un instrument pour le novice afin qu'il puisse développer progressivement son expertise et accroître ses espaces d'autonomie et sa polyvalence.

A la suite de cette introduction, l'article est organisé en 5 parties. Dans un bref parcours historique, nous reprenons les premières tentatives du projet ESPRIT de l'Union Européenne, dans le cadre duquel des technologies anthropocentriques réunissant ingénieurs et scientifiques sociaux proposent d'expliquer les échecs par l'absence d'une théorie de l'activité fondée empiriquement (partie 2). Ceci nous mène directement à l'intérêt de la théorie du cours de l'action pour le développement d'artefacts technologiques appropriés et dont les opérateurs peuvent s'approprier afin d'étayer "le caractère anthropologiquement constitutif de la technique" (Stiegler, 2018). À propos de la technique dans le cadre du PRCA, cette étude se penche plus spécialement sur le programme technologique, présenté aujourd'hui sous la forme d'une « ingénierie de situations de travail ", à la recherche de l'articulation entre les artefacts matériels, l'organisation du travail et la formation (partie 3). L'effectivité et la richesse de ce programme sont attestées par les produits de formations, d'automation et d'opération collective $\mathrm{H}-\mathrm{H}-\mathrm{M}$ issus de l'intervention dans les PCHs (partie 4). En guise de conclusion, nous mettons l'accent sur notre contribution au pôle technologique du PRCA et nous la renvoyons vers le débat actuel sur l'industrie 4.0 qui révèle le manque de mémoire historique de l'ingénierie et insiste sur la répétition du mythe de l'usine sans hommes, question déjà présente au sein de nos débats sur le projet anthropocentré depuis les années 1970.

\section{Impasses des projets sociotechniques et anthropocentrés}

16 Sur la lancée des mouvements sociaux et syndicaux des années 1960, diverses approches ont surgi comme alternative à la conception technocentrique de l'ingénierie. Dans le cadre de ce mouvement, nous pouvons rappeler le sociotechnical system design (STSD) (Eijnatten, 1993), dont la création remonte aux travaux d'Emery et Trist de l'Institut Tavistok à la fin des années 1940 ; le work-oriented design (Ehn, 1988) ; le projet anthropocentré (Brödner, 1990 ; Brödner, 1993 ; Corbett, Rasmussen, \& Rauner, 1991) ; la social shaping of technology (Corbett et al., 1991) ; l'activity oriented design (Engeström, 1987 ; Nardi, 1997), sans oublier de mentionner les approches critiques comme la construction sociale de la technologie (Bijker, Hugues, \& Pinch, 1989). Ces approches ont un intérêt commun : celui d'intégrer les hommes et les artefacts techniques par le biais d'un projet englobant au moins trois éléments ou aspects des systèmes H-M : 1) les artefacts proprement dits ; 2) le travail, c'est-à-dire la formation des travailleurs ou l'organisation du travail; 3) les interfaces hommes-machines. Ces approches sont également similaires du point de vue méthodologique, car elles adoptent des méthodes participatives plus ou moins approfondies.

17 Ces dernières sont toujours débattues à ce jour, car elles sont difficilement diffusables même lorsqu'elles produisent des résultats supérieurs à ceux de projets conventionnels technocentrés. Cette situation peut s'expliquer par les antagonismes politiques et les stratégies entrepreneuriales, ${ }^{3}$ mais, sans minimiser ces déterminations politico-sociales 
importantes, il existe des problèmes non résolus au niveau même du projet des systèmes H-M. Les expériences de coopération entre les sciences sociales et les ingénieries ne se sont pas suffisamment consolidées pour constituer de fait un "paradigm that changed the workplace ", pour reprendre le titre optimiste de l'ouvrage de Frans Eijnatten (1993) sur le STSD. Nous pensons que ces expériences étaient dépourvues de théorie consistante sur l'activité humaine afin de pouvoir orienter les projets d'ingénierie, concevoir des interfaces et projeter l'organisation du travail de façon intégrée. Cette hypothèse peut se vérifier en reprenant certaines expériences emblématiques des années 1970 et 1980.

Corbett, Rasmussen et Rauner (1991) ont coordonné, dans le cadre du programme ESPRIT, des équipes multidisciplinaires dans trois pays européens (Angleterre, Danemark et Allemagne) dans le but de développer un projet CIM (Computer Integrated Manufacturing) anthropocentré. Durant ce programme, ils ont créé l'expression « social shaping of technology" afin de promouvoir le dialogue entre les ingénieurs et scientifiques sociaux qui participaient au projet. Avec des équipes de travail dans trois pays différents et chacun étant responsable d'un sous-projet, ils ont pu expérimenter des dispositions sociales différentes et les mettre en lien avec des résultats différents plus ou moins proches des principes du design anthropocentré. Pour leur élaboration, les projets ont travaillé trois aspects avec plus ou moins d'intensité : projet de l'artefact technique, design des interfaces et projet organisationnel (organisation du travail et formation). Dans le bilan final de ces expériences, les auteurs ont officialisé le concept de «crossing the border " grâce auquel ils ont tenté de généraliser les résultats en termes de processus social de conception, plus comme proposition méthodologique et moins comme résultat effectif des expériences, qui n'ont pas atteint les objectifs initiaux.

En ce qui concerne la ligne allemande, Böhle et Milkau (1988) ont démontré comment est effectué le rapprochement de l'activité humaine avec le projet technique en proposant le concept de l'activité subjectivante ${ }^{4}$. Bien qu'il s'agisse d'une étude de sociologie du travail essentiellement analytique, les auteurs en ont tiré des conclusions pour le projet de systèmes automatisés permettant le développement de l'activité subjectivante avec de nouvelles configurations et distributions de fonctions entre l'homme et la machine. Ces propositions englobent aussi la formation et l'organisation du travail, y compris la coopération entre les opérateurs des machines et les concepteurs. L'objet de l'analyse était l'introduction de machines d'usinage CNC (Commande Numérique) pour remplacer des machines manuelles où les opérateurs avaient un contact direct avec la machine et le matériel usiné. Tout le corps de l'opérateur était engagé dans la réalisation du travail qui faisait appel aux sens du toucher, de l'ouïe, du goût, de la vision et de la proprioception. Avec l'introduction de la $\mathrm{CNC}$, une distance a été créée empêchant le contact direct avec la machine et empêchant même la visualisation. Les auteurs montrent toutefois comment les opérateurs de systèmes automatisés arrivent à reconstituer l'activité subjectivante avec la perception de bruits significatifs. Sans cette perception, ils ne réussissaient pas à anticiper la panne d'un outil ou son usure. On peut donc conclure que la contribution du corps n'est pas uniquement nécessaire pour opérer des machines manuelles, ce à quoi on s'attendait, mais également dans des systèmes automatisés. Il nous faut donc réfléchir sur les conditions de développement de l'activité subjective pour opérer des machines $\mathrm{CNC}$, surtout lorsque l'expérience avec des machines manuelles tend à devenir plus rare pour les nouvelles générations d'opérateurs qui n'ont pas vécu la transition. Dans le cadre de notre discussion, les recommandations pratiques pour 
résoudre les problèmes mis en évidence par le projet du travail sont intéressantes, car elles touchent à la formation, à l'organisation du travail et aux machines.

La reconnaissance de l'activité subjectivante conduit à déconseiller d'allouer un opérateur à plusieurs machines, car même les machines automatiques sont uniques et exigent un savoir intime de l'opérateur pour dominer «sa» machine. Occuper d'éventuels « temps morts » par d'autres tâches empêche l'opérateur d'accompagner sa machine et de percevoir les variations de bruit qui indiquent l'usure d'un outil ou une autre modification du processus (acier, fluide réfrigérant, etc.). La pratique du job rotation est également déconseillée, car elle complique le développement de cette perception raffinée de la machine. Avec des protections, le point d'usinage est quasi inaccessible pour l'opérateur qui ne peut que compter sur son ouie pour suivre le processus. Permettre un accès ou une visualisation directe, surtout lorsque la production d'un nouveau lot débute, aiderait à stabiliser le processus pendant ce moment critique.

21 En termes de logique de programmation, les opérateurs ont également besoin ici de réaliser des interventions qui sont normalement interdites lorsque l'on adopte une programmation centralisée :

Au niveau de l'organisation du travail, les marges d'expérimentation sur les machines doivent être intégrées comme des composantes du comportement du travail. Comme nous l'avons montré, c'est pour les ouvriers une condition importante pour un commerce sûr et optimal avec les machines. Le principe suivant doit pouvoir être vérifié, y compris sur les machines CNC: «celui qui n'essaie rien et qui n'ose rien n'utilisera jamais la machine de forme optimale, il n'aura aucune assurance dans sa maîtrise. » (Böhle \& Milkau, 1988, p. 143).

22 Les machines manuelles, l'autonomie et le savoir tacite des opérateurs, véritables artisans de l'usinage, étaient connus et valorisés tandis que, dans les systèmes automatisés, les opérateurs sont encore considérés comme de simples «appuyeurs de boutons » et n'auraient que très peu à échanger avec les programmeurs. Toutefois, il devrait également y avoir ici une coopération entre les services de programmation et de production pour établir un dialogue au sens plein du terme :

«Mais il faut aussi que soient créées les conditions qui permettent aux opérateurs, $\mathrm{du}$ fait de leur savoir particulier et de leur expérience pratique avec les machines, de faire valoir certaines propositions et critiques par rapport à la programmation et à la gestion de la production. Ceci n'est que trop peu pris en compte actuellement. Jusqu'à présent, la coopération n'est possible que lorsque l'initiative en vient du service de programmation ou de celui de la gestion de la production » (Böhle \& Milkau, 1988, p. 144).

Pour compenser le défaut des sens nécessaires à la reconstruction de l'activité subjectivante, les opérateurs de CNC développent ce que les auteurs appellent « une sensibilité abstraite » afin de suivre le processus d'usinage, de réussir à anticiper les problèmes et d'optimiser le fonctionnement des machines. Dans l'impossibilité d'avoir un feedback immédiat de l'usinage par les sens et le corps, les opérateurs doivent se représenter le fonctionnement de la machine :

«Ces représentations ne sont pas le fruit de l'imagination. Elles reposent sur des expériences réelles transposées et appliquées à des situations dans lesquelles elles ne sont plus possibles de la même façon. Elles présentent des caractéristiques d'une "sensibilité abstraite". Elles sont "sensibles" parce que, comme dans le cas d'expériences et de perceptions sensorielles réelles, elles sont développées avec et sur la base des différents sens. Ceci est caractérisé par le fait que la représentation des opérations est "imagée" et que les ouvriers "voient" les déroulements 
représentés; de façon analogue, il s'établit également des associations avec le commerce tactile, avec l'objet: on croit avoir le matériau dans la main, le sentir, bien qu'on ne le touche pas réellement. Une telle perception sensible est "abstraite" parce qu'elle est détachée, "abstraite" des déroulements et des objets concrets, de même que dans la pensée conceptuelle, on s'abstrait des réalités concrètes. »

(Böhle \& Milkau, 1988, p. 114)

L'expression "sensibilité abstraite» peut sembler étrange, car elle combine des représentations abstraites, des images avec des perceptions sensibles, mais il est important de reconnaitre les phénomènes auxquels elle renvoie. Dans les cas empiriques présentés ci-dessous, nous verrons que ces impressions subjectives apparaissent sous forme d'abstractions telles que « sentir le canal » lorsque l'opérateur d'une PCH ajuste le débit d'eau pour optimiser la génération d'énergie. De la même façon, on peut également vérifier l'émergence de représentations ou images, jusqu'alors non disponibles, avec l'aide d'objets intermédiaires développés pour orienter la formation des opérateurs de salles de contrôles à distance. Bien que ces représentations soient reconnues dans les approches anthropocentrées, elles ne sont pas encore produites de forme organisée ou par des moyens systématisés. L'approche du cours de l'action réussit à surmonter ces difficultés grâce à sa compréhension de l'ergonomie en tant que technologie.

\section{Théorie du cours de l'action et programme technologique}

25 La « théorie du cours de l'action » (TCA) s'est développée dans le cadre d'un programme de recherche empirique en anthropologie cognitive et technologique. En termes pratiques, son objectif central est de fournir des descriptions suffisamment détaillées de l'activité (dans ce sens, elle est définie comme une praxéologie empirique) qui puissent créer des modèles pertinents de conception pour les concepteurs. Dans l'introduction de Méthode Réfléchie, Theureau (2009), en présentant le programme de recherche philosophique associée aux deux programmes cités antérieurement, annonce la publication future d'ouvrages plus spécifiques et, entre autres, des études pour spécifier le programme de recherche technologique. Dans cet article, nous nous basons surtout sur l'ouvrage collectif (Theureau \& Jeffroy, 1994) dans lequel les contributions du cours de l'action au projet de situations de travail informatisées sont plus développées et où il est possible de situer la question du passage de la description de l'activité pour les recommandations du projet. Bien qu'elle n'ait pas été appropriée par l'ensemble des ergonomistes, comme c'est le cas dans d'autres domaines, comme par exemple pour la conception de systèmes informatisés et l'éducation, notamment dans le cas des STAPS (Sciences et Techniques des Activités Physiques et Sportives), la théorie du cours de l'action est, à notre avis, le programme le plus avancé et le plus ambitieux de théorisation et de formalisation d'une praxéologie empirique dans le champ de l'ergonomie. Nous n'affirmons pas que tous les problèmes aient été résolus, comme le reconnaît Theureau (1992) en attribuant l'adjectif « baroque » à cette théorie. Dans cet article, nous discutons spécifiquement comment faire avancer le programme technologique avec la description d'un cas (partie 5) comportant des interventions intégrées dans trois domaines: la formation, les spécifications d'automation et l'organisation du travail (opération conjointe salle de contrôle/terrain). 
26 Nous partons du principe général, associé à d'autres approches inspirées par la théorie de l'activité instrumentale de Vygotski, que le développement humain passe nécessairement par un projet approprié des situations de travail, c'est-à-dire qu'il faut reconnaître le « caractère anthropologiquement constitutif de la technique », comme le propose Stiegler (2018) qui identifie trois types de genèse de l'être humain à un moment donné :

- « la genèse ayant pour produit la mémoire génétique : la transmission génétique ;

- l'épigenèse ayant pour produit la mémoire nerveuse : ce qui vient de l'apprentissage et de la formation au cours d'une vie;

- l'épi-phylogenèse ayant pour produit la mémoire technologique (associant technique et langage, et plus généralement tout ce qui fait signe) : ce que chaque génération trouve comme déjà là construit par la technique. » (Theureau, 2004, p. 22).

Cette imbrication du développement humain et technique a, selon Theureau, d'importantes conséquences pour la conception ergonomique qui, initialement pensée en termes «de support», doit être modifiée en termes "d'appropriabilité de la technique ». En d'autres termes, "penser à la conception de l'ensemble de manière à aider effectivement l'homme en situation, mais également et surtout, contribuer à produire une humanité désirable ou, pour le moins, un aspect inhérent à cette humanité désirable » (Theureau, 2004).

Mais comment traduire les descriptions de l'activité située, toujours contextualisée, dynamique et contingente en recommandations de projet qui, à la fin du compte, opère une "congélation » ou réification du travail ? Les notions du cours de l'action sont dynamiques et concernent des réalités transitoires « car elles renvoient à des coupures temporelles plus ou moins importantes du flux (Theureau \& Jeffroy, 1994, p. 245). Néanmoins, en ce qui concerne au moins les interprétants acquis, on peut constater une certaine stabilité des types :

«L'hypothèse de la composition des interprétants acquis par des types et des relations entre types implique une certaine stabilité temporelle et situationnelle entre les différentes sortes de types et relations entre types » (Theureau \& Jeffroy, 1994, p. 222).

«Il est d'ailleurs remarquable que nombre d'études d'ergonomie et psychologie du travail emploient le mot "schéma" à propos de "règles" plus ou moins locales, morcelées, changeantes, repérées dans l'analyse de données sur l'activité, sans définir une quelconque architecture de schémas enchâssés, c'est-à-dire, sans employer la "théorie des schémas" ». (Theureau \& Jeffroy, 1994, p. 227).

Pour le processus de conception, ce sont surtout les modèles de transformation produits à partir des descriptions et analyses du cours de l'action qui nous intéressent. Dans ce sens, la différence entre les "cours de l'action types " et les "schémas " souligne les avantages des premiers quant aux relations qui peuvent s'établir entre les modèles et les situations projetées, à savoir :

«Les types et relations entre types mis en évidence dans les études empiriques du cours de l'action sont plus locaux, morcelés, changeants. »

« De même, les relations entre les types apparaissent plus variées que les relations d'enchâssement. D'une part, un type ou une relation entre types peut entrer en une relation d'analogie avec d'autres par des jeux de ressemblances et différences très variés. (...) D'autre part, un type ou une relation entre types peuvent être connectés avec d'autres par chacun ou une partie des évènements, actions et communications types qui les composent. » (Theureau \& Jeffroy, 1994, p. 227). 
Ces caractéristiques dynamiques des types reconnus ici, au moment de décrire l'activité, auront a fortiori de plus grandes conséquences lorsqu'il s'agira de fournir des modèles de l'activité aux concepteurs. Le cours de l'action est l'objet de descriptions détaillées qui permettent de formaliser des modèles d'analyse à des niveaux différents, globaux ou locaux, d'organisation de l'activités.

«Le modèle de construction global est un ensemble cohérent de structures significatives qui dévoilent des régularités sous-jacentes au développement des cours d'action comme un tout. » (p. 98)

«Le modèle de construction local est un modèle d'analyse du surgissement à chaque instant d'une ou autre unité significative élémentaire de cours d'action, c'est-à-dire d'une action, communication, interprétation ou focalisation » (p. 99)

«Les modèles des transformations (synchroniques et diachroniques) du cours d'action relient les caractéristiques de l'organisation intrinsèque du cours de l'action (engendrement, composition) avec des contraintes extrinsèques (de l'état des acteurs, de leur situation, et de leur référentiel). Nous le nommons ainsi, car ils permettent de passer d'un cours d'action (d'un acteur donné, dans une situation donnée, à un moment donné) à un autre. Ces modèles des transformations du cours d'action peuvent être développés en modèles des transformations du cours d'action et de ses effets (concernant l'état des acteurs, leurs situations et leur référentiel). » (Theureau \& Jeffroy, 1994, p. 99)

Ces principes de traduction des analyses dynamiques du cours de l'action, en termes de recommandations de projet, ont été annoncés depuis les premières formalisations de la TCA :

«Les notions théoriques que nous avons exposées permettent de construire des modèles des cours d'action étudiés. (...) Ce sont des modèles du cours d'action et non des modèles de l'utilisateur (c'est-à-dire, soit un mécanisme qui décrit son fonctionnement, soit sa représentation du fonctionnement du système. Ces modèles sont de différentes sortes selon la nature des cours d'action étudiés. Par exemple, pour un travail de saisie, ils mettent en évidence les COA-TYPES organisant la saisie (Pinsky \& Theureau, 1987); pour les activités de résolution de problèmes d'exploitation de micro-ordinateurs, ils décrivent les raisonnements menés par les opérateurs (Jeffroy, 1987). » (Pinsky, 1992, p. 117-8).

D'autres difficultés méthodologiques doivent toutefois être surmontées pour construire cette théorie «baroque » de l'activité du travail afin de passer de la description globale ou locale aux modèles opérationnels dans la perspective des concepteurs. Dans le cadre de la TCA, cette fonction est remplie par les hypothèses concernant la transformation du cours de l'action.

"Ces hypothèses mettent en lien les caractéristiques de l'organisation intrinsèque (ou construction) des cours de l'action et les limites et effets extrinsèques (concernant l'état des acteurs, leur situation et leur culture). » (Theureau \& Jeffroy, 1994, p. 106)

«Ces hypothèses de transformation du cours de l'action peuvent être systématisées grâce à l'élaboration de ce que nous nommons les modèles de transformation. (...) De tels modèles de transformation sont intéressants, car ils débouchent directement sur des scénarios et modèles de conception» (Theureau \& Jeffroy, 1994, p. 107).

Nous arrivons maintenant au problème du passage entre l'analyse et la formalisation de recommandations que nous avons identifié comme étant la question centrale de toutes les approches de l'ergonomie de la conception ${ }^{6}$. Cependant, comme le reconnaissent les auteurs, si les hypothèses de transformation existent dans toutes les études de conception centrées sur le cours de l'action, « des modèles de transformation n'ont été 
que rarement produits " (Theureau \& Jeffroy, 1994, p. 107). Le cas présenté ci-dessous explicite surtout les representamens qui font partie des jugements tacites dans les processus de décision et de résolution des problèmes par les opérateurs de terrain des $\mathrm{PCH}$. Ces processus sont organisés en modèles relativement simples d'activité, tels que les arbres des causes qui, néanmoins, sont des instruments efficaces pour organiser la formation, l'organisation du travail collectif de co-opération de la salle de contrôleterrain et pour orienter le projet des systèmes automatisés. Les representamens sont l'une des composantes du signe tétradique (Figure 1) qui est un concept élémentaire de la TCA développé à partir du signe triadique de Peirce. L'activité est décrite comme un enchainement de signes tétradiques composés, hormis les representamens par l'Instance de Référentiel et par l'Ouvert (antérieurement nommé Objet). L'action de l'acteur en situation apparaît comme une conséquence des interrelations entre l'expérience accumulée de l'acteur et ce qui lui vient à l'esprit (Instance de Référentiel), ce que (dans le monde) lui est demandé sur le moment (representamen) et les options qui se présentent à lui (Ouvert) à partir de la combinaison des composantes du signe. En agissant, une nouvelle situation (en tant que nouveau representamen, une instance de référentiel modifiée et de nouveaux possibles) est configurée pour l'acteur et la dynamique d'activité est alors dévoilée comme un enchainement de signes et processus de transformation des éléments constitutifs.

Figure 1 : Signe tétradique (Theureau, 2014).

Figure 1 : Tetradic signe (Theureau, 2014)

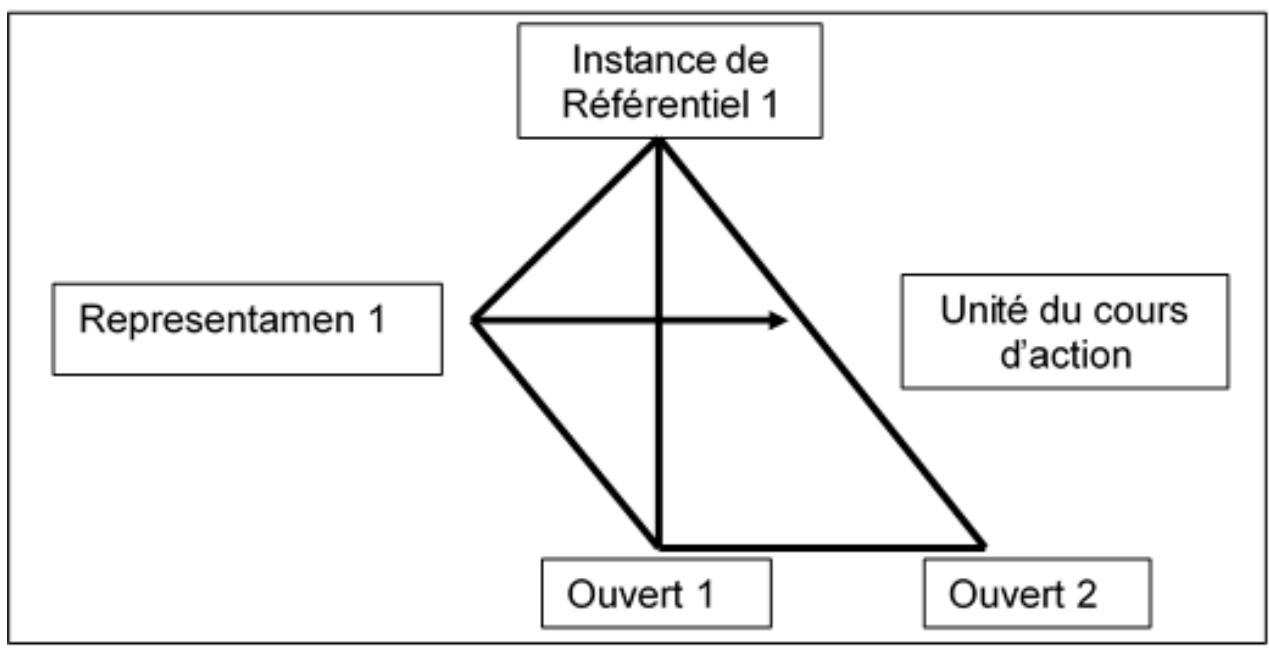

\section{Modèles de transformation en automation de $\mathrm{PCH}$}

L'activité analysée est une opération locale de trois $\mathrm{PCH}$ « en cascade » approvisionnés par un barrage d'accumulation. L'eau du barrage est contrôlée par une vanne et arrive à l'usine par la rivière principale; dans chaque usine, il y a un petit barrage de contention et des comportes pour orienter l'eau de la rivière dans un canal d'adduction. Le cours d'eau principal et les canaux sont alimentés sur toute leur longueur par des rivières adjacentes. Pour ces rivières, c'est la même structure de barrage-comporte qui est utilisée. Le complexe énergétique peut être mieux compris à l'aide de la Figure 2 ci-dessous. 
Figure 2 : Complexe énergétique des $\mathrm{PCH}$.

Figure 2: SHPP energy complex
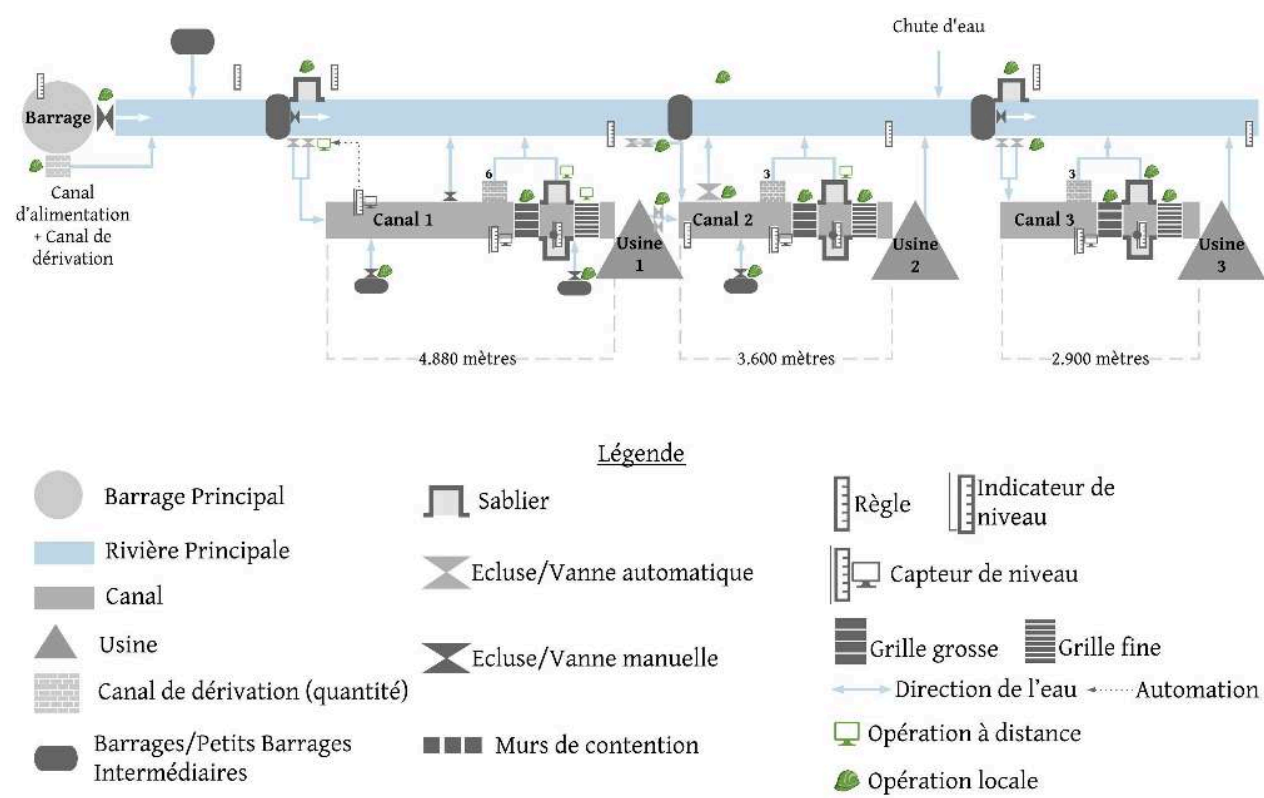

Source : Situated Consultoria e Pesquisa de vidéos et photos qui ont servi de support aux entretiens en autoconfrontation. La construction des arbres des causes requiert l'utilisation de divers symboles qui attirent l'attention sur les points principaux identifiés durant les analyses de chaque activité et synthétisent les éléments essentiels qui sont tacites pour les opérateurs expérimentés. Nous présentons dans le Tableau 1 une description résumée de chaque symbole et leur référence dans le cadre de la TCA. 
Tableau 1 : Description des principaux symboles utilisés dans les arbres de jugement. Table 1: Description of the main symbols used in the judgment trees

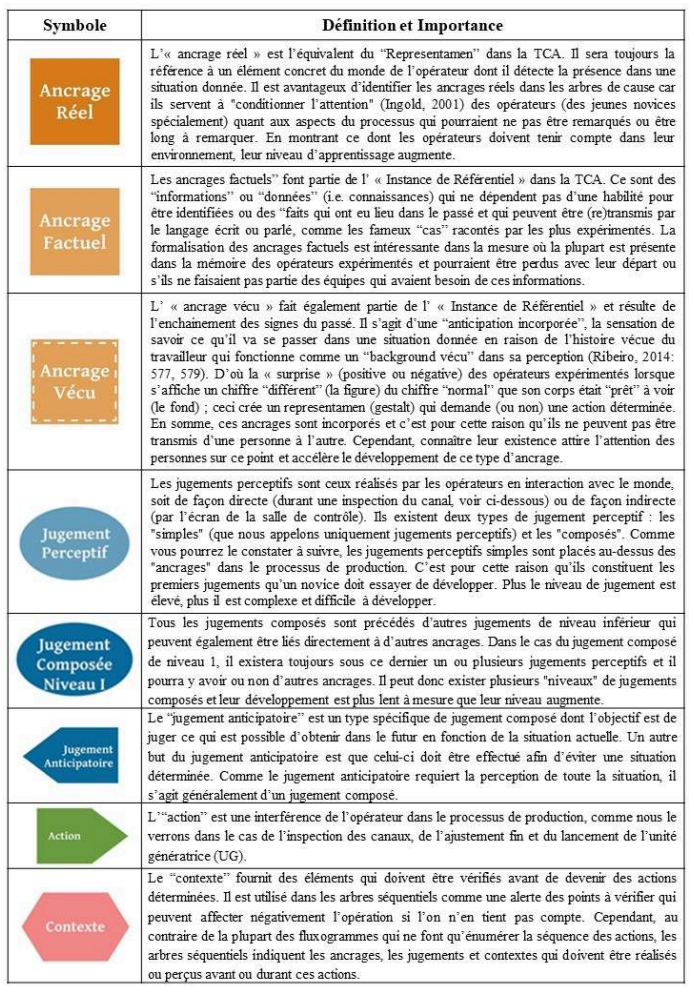

En relation au vocabulaire adopté dans la TCA, l'utilisation du terme « ancrage » a été adoptée pour trois raisons. La première pour faciliter la compréhension et l'utilisation des arbres des causes par les usagers : opérateurs et ingénieurs de l'entreprise. Avec cette terminologie, il était aisé d'expliquer à tous que les ancrages étaient ceux qui servaient à réaliser ou « fixer » les jugements réalisés par les travailleurs expérimentés. En outre, la division en trois types d'ancrage montrait des aspects qui pouvaient être respectivement identifiés (ancrages réels), formalisés (ancrages factuels) ou développés (ancrages vécus). La seconde raison est que les ancrages factuels et virtuels distinguent deux aspects de nature différente de l'instance de référentiel. Finalement, bien que les ancrages réels soient issus des representamens présents dans les cours de l'action analysés, lorsqu'ils sont tous placés dans un arbre des causes (voir Figure 4), ils ne constituent pas à proprement dit des « representamens » car ils sont « en dehors » de l'action.

Comme nous l'avons informé préalablement, trois produits ont été créés durant l'intervention. Ces derniers ont fourni trois types de contribution plus anthropocentrés au projet de systèmes H-H-M :

1. Création « d'objets intermédiaires de formation " grâce à la systématisation du savoir tacite des opérateurs expérimentés et sa formalisation dans des « arbres des causes » associés à des activités spécifiques et des "arbres séquentiels» qui présentent des jugements tacites préalables aux séquences d'action. Ces dispositifs facilitent l'échange d'expériences entre les spécialistes et l'apprentissage des novices; par «novices ", il faut entendre les travailleurs sans expérience et les travailleurs expérimentés qui n'ont œuvré que dans l'une des PCH, mais qui vont travailler dans une autre unité ;

2. Développement d'un "modèle analytique pour l'automation anthropocentrée et appropriable» basé sur des representamens caractéristiques des activités critiques 
analysées qui opérationnalise les principes de co-opération et d'appropriabilité des technologies automatiques ;

3. Développement d'un «modèle analytique de co-opération: opération locale versus opération à distance » qui agit comme un «objet intermédiaire de co-opération » entre opérateurs de terrain et à distance allant au-delà de la division de fonctions élaborée initialement, selon laquelle les opérateurs de la salle de contrôle prenaient les décisions et les autres opérateurs ne faisaient que les exécuter, pour contribuer à transformer le « collectif de travail » en un « travail collectif» (Assunção, 1998 ; Caroly, 2012).

\subsection{Objets intermédiaires de la formation : arbres des causes et séquentiels}

Le premier produit répondait à la demande initiale de formalisation (dans la mesure du possible) des savoirs tacites des opérateurs expérimentés afin de préserver les savoirs spécifiques de chaque usine, de faciliter la polyvalence, d'aider à la formation des novices et d'accélérer la formation des opérateurs à distance (par des visites guidées). Ceci a été exécuté de deux manières différentes par des arbres des causes et séquentiels. Comme exemple du premier cas, nous débattrons l'activité d'inspection des canaux d'adduction, choisie pour son caractère critique. Dans le second cas, nous présentons l'arbre séquentiel de l'activité de lancement de l'Unité Génératrice (UG).

\section{Arbres des causes}

Les canaux d'adduction du Complexe Energétique analysé mènent l'eau du lit normal de la rivière aux conduites forcées des usines sur un parcours de $11 \mathrm{kms}$. Les conditions structurelles de ces infrastructures sont contrôlées tous les deux ou trois jours par les opérateurs locaux - même pour les parties difficiles d'accès - afin de vérifier s'il existe des fuites ou d'autres indices pouvant conduire à des ruptures de structure sur des canaux de plus de 60 ans (Figure 3).

Figure 3 : Le parcours depuis le barrage qui jette l'eau dans la rivière par la vanne, le passage par le canal d'adduction et le conduit forcé jusqu'à l'arrivée dans la PHC.

Figure 3: The path from the dam pouring water into the river through its valve, the passage through the adduction channel and the penstock until arrival at the SHPP

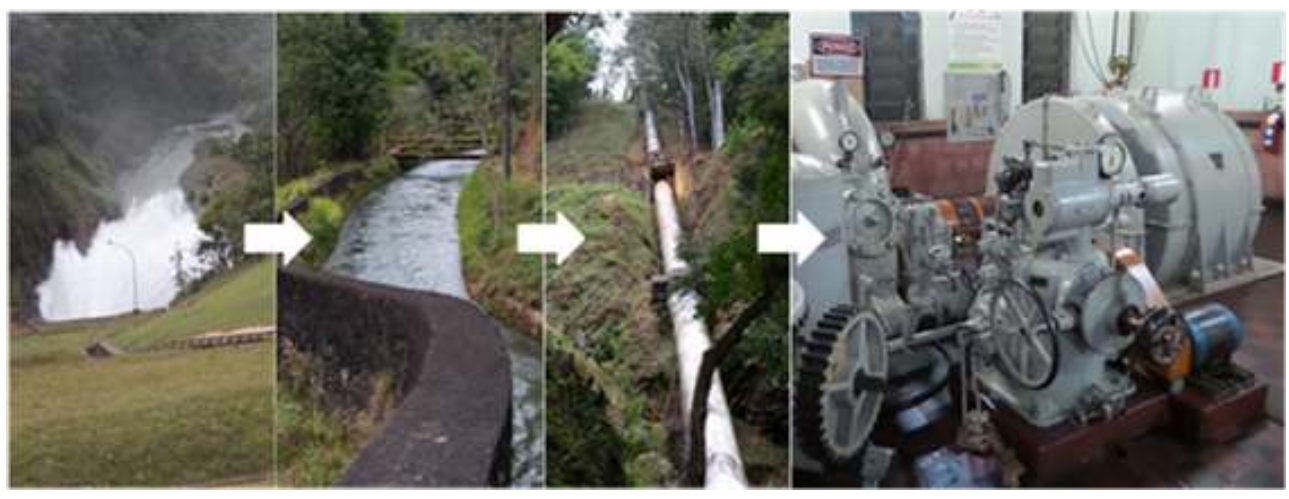

Source : Situated Consultoria e Pesquisa

L'inspection augmente la sécurité des routes, des communautés riveraines et de l'environnement et prévient les accidents et arrêts dus à des incidents de ruptures qui ont des impacts sur la productivité de l'usine et sur « l'agrément social » de l'entreprise 
octroyé informellement par la communauté avoisinante. Vu son importance, cette activité a été choisie pour accélérer l'apprentissage des novices pour identifier les problèmes et également pour développer le jugement de leur criticité (importante, moyenne ou faible criticité). Lorsque les opérateurs de terrain trouvent des indices de fuite ou de rupture, l'inspection est immédiatement effectuée. C'est également le cas pour une fuite ou une fissure de faible criticité: ils augmentent la fréquence de l'inspection durant la semaine afin de vérifier l'évolution de la fissure. Le jugement de «criticité de la fuite ou de la rupture» est donc l'un des principaux jugements à développer pour garantir la protection des canaux, car, selon sa criticité, l'opérateur agit différemment avec un « sens approprié de l'urgence " pour résoudre ou minimiser de possibles impacts dans chaque cas.

La Figure 4 présente l'arbre des jugements de l'inspection des canaux, les jugements sont représentés par des ellipses bleues et les actions par des ellipses vertes. Dans le cadre de la discussion, la structure et le contenu général des arbres sont plus importants que les détails de chaque action ou jugement qui sont peu lisibles dans les figures. En prenant l'exemple du jugement de "criticité de la fuite ou de la rupture ", on peut vérifier qu'il existe quatre ensembles d'informations et de jugements (représentés par les flèches numérotées qui aboutissent à ce jugement) afin qu'ils puissent être réalisés : (1) le jugement de la «nature du problème »; (2) l'endroit de la fuite ou de la rupture; (3) les ancrages factuels et (4) le contexte de la fuite ou de la fissure.

Figure 4 : Arbre de jugements de «Criticité de la fuite ou rupture».

Figure 4: Tree of Judgments of "Criticality of Leak or Breakage"

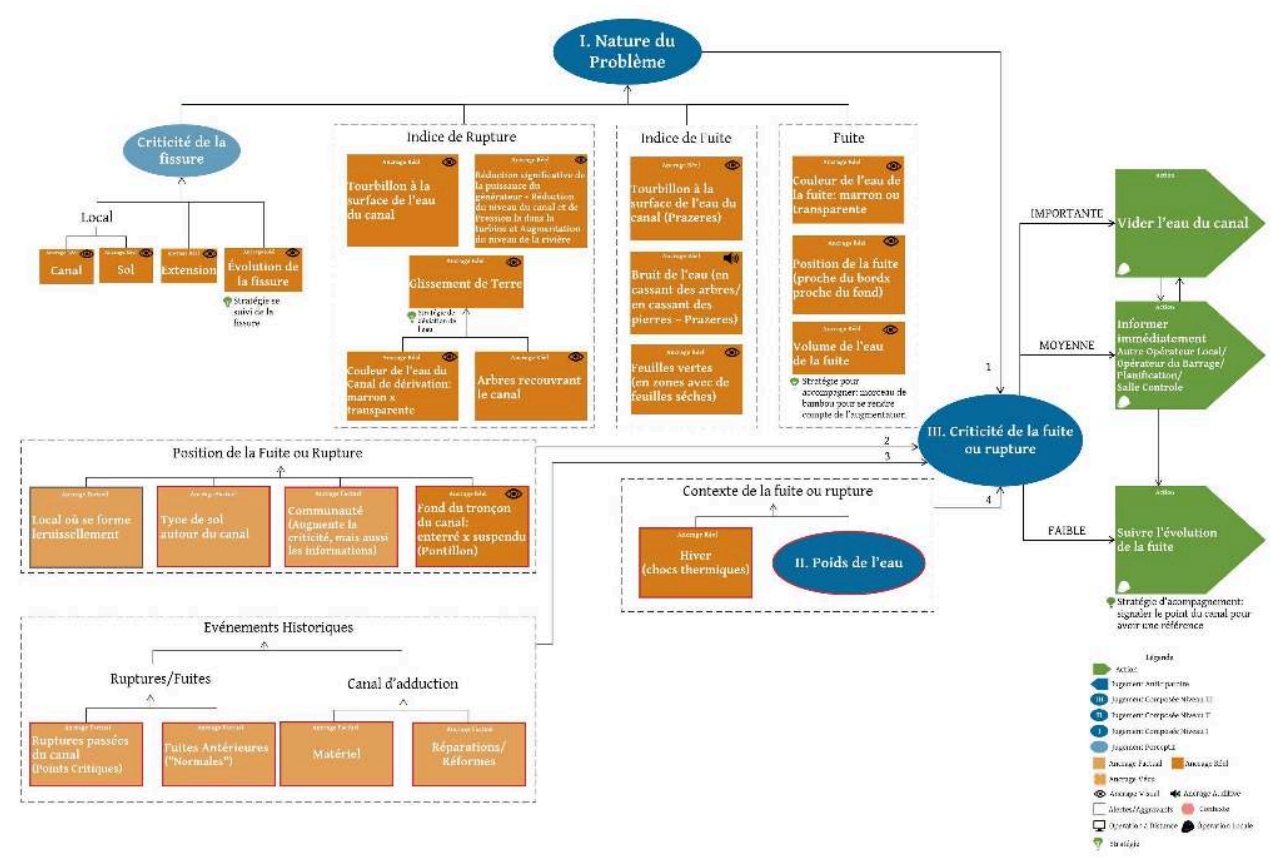

Source : Situated Consultoria e Pesquisa

Pour évaluer la criticité d'une condition donnée, l'opérateur tient compte en premier lieu de la nature du problème en question (voir haut de la Figure 4). Le problème est-il un indice de rupture? S'agit-il d'un indice de fuite ou déjà d'une fuite? Pour identifier chaque cas, il y a des ancrages réels qui y sont associés. Par exemple, les ancrages 
"d'indice de rupture " sont tous réels et visuels et indiquent si la situation identifiée peut être à l'origine de la rupture du canal (voir Tableau 2 pour la description de certains ancrages vérifiés qui définissent un «indice de rupture»). En outre, l'opérateur peut également trouver des fissures, accompagnées ou non de fuites. Lorsqu'elle existe, la criticité de la fissure est un «jugement perceptif » simple (basé exclusivement sur des ancrages réels) qui aide à identifier la nature du problème. C'est un important point de départ pour que l'opérateur puisse juger la criticité de la situation. Par contre, le jugement de la "nature du problème" est un jugement composé de niveau 1 qui prend en compte 11 ancrages réels situés en dessous dans le graphique et également du jugement perceptif simple de la « criticité fissure » (si c'est le cas).

Tableau 2 : Ancrages réels de "l'Indice de rupture ».

Table 2: Real anchors of "Break Indication"

\begin{tabular}{|c|c|}
\hline Ancrage & Description \\
\hline $\begin{array}{l}\text { Tourbillon à la superficie de } \\
\text { l'eau du canal }\end{array}$ & $\begin{array}{l}\text { Les tourbillons indiquent qu'il y a une grande fuite dans le fond du canal car } \\
\text { un grand volume d'eau est en mouvement. }\end{array}$ \\
\hline $\begin{array}{l}\text { Glissement du flanc de } \\
\text { coteau }\end{array}$ & $\begin{array}{l}\text { Les glissements de terrain peuvent impacter gravement la structure du canal. } \\
\text { Dans le cas de l'usine } 3 \text {, où le sol autour du canal est rocheux, les } \\
\text { glissements, bien que rares, peuvent être très graves et ils demandent des } \\
\text { interventions plus longues pour retirer le matériel du canal. Un glissement } \\
\text { peut être identifié de deux façons : dans l'usine lorsque l'eau du canal } \\
\text { d'écoulement est boueuse ; et dans le canal même, avec la végétation qui } \\
\text { commence à "envahir" une partie des parois latérales ; ou encore lorsque des } \\
\text { éléments de la paroi commencent à tomber. }\end{array}$ \\
\hline $\begin{array}{l}\text { (1) Couleur de l'eau dans le } \\
\text { canal d'écoulement }\end{array}$ & $\begin{array}{l}\text { En cas de glissement et lorsque de nombreux éléments tombent dans le canal, } \\
\text { l'eau du canal d'écoulement de l'usine peut être boueuse, ce qui indique } \\
\text { qu'elle contient de la terre. Cette différentiation est plus commune durant la } \\
\text { période sèche lorsque l'eau est plus propre. Durant la saison des pluies et } \\
\text { quand la couleur de l'eau est plus marron, il est plus difficile de visualiser cet } \\
\text { ancrage. }\end{array}$ \\
\hline $\begin{array}{l}\text { (2) Végétation "recouvrant" } \\
\text { le canal }\end{array}$ & $\begin{array}{l}\text { En parcourant la longueur du canal, les opérateurs identifient s'il existe de la } \\
\text { végétation sur les parois latérales du canal qui commencent d'ailleurs à } \\
\text { "recouvrir" certaines parties de la structure. Cet ancrage est un indice de } \\
\text { glissement de paroi à cet endroit. }\end{array}$ \\
\hline
\end{tabular}

Un autre ensemble d'éléments de base pour évaluer la criticité d'une fuite ou d'une rupture est l'« endroit de la fuite ou de la rupture ». Dans cette situation, il existe des ancrages factuels et réels (par exemple, le fond du tronçon et le type de sol) qui augmentent le potentiel de fuites et de ruptures entre autres (par exemple, la présence de communautés avoisinantes) qui aggravent la situation. La description des ancrages de l'« endroit de la fuite ou de la rupture » se trouve dans le Tableau 3 à suivre. 
Tableau 3 : Ancrages de l'« endroit de la fuite ou de la cassure ». Table 3: Anchors of the "Location of the Leak or Break"

\begin{tabular}{|l|l|}
\hline \multicolumn{1}{|c|}{ Ancrage } & \multicolumn{1}{c|}{ Description } \\
\hline $\begin{array}{l}\text { Parcours des eaux de pluie } \\
\text { (Gouttières) } \\
\text { (Pluies) }\end{array}$ & $\begin{array}{l}\text { Durant les inspections en période pluvieuse, il y a de l'eau dans le sol autour } \\
\text { du canal et des ruissellements peuvent se former lorsqu'il pleut. Les } \\
\text { opérateurs réussissent à différencier l'eau provenant de la pluie ou d'une fuite } \\
\text { car ils connaissent les endroits où les eaux de pluie passent et ceux où il y a } \\
\text { des gouttières (petits déversoirs d'écoulement). Ils savent donc où se trouvent } \\
\text { les grands volumes d'eau provenant des pluies et où les fuites ne devraient } \\
\text { pas s'écouler. Il s'agit donc d'un ancrage factuel. }\end{array}$ \\
\hline $\begin{array}{l}\text { Type de sol autour du canal } \\
\text { (Perméable) }\end{array}$ & $\begin{array}{l}\text { Plus le sol est perméable autour du canal, plus les chances de fuites et } \\
\text { cassures sont importantes. Il s'agit donc d'un ancrage factuel. }\end{array}$ \\
\hline Communauté & $\begin{array}{l}\text { La présence de communautés adjacentes augmente la criticité d'une fuite ou } \\
\text { d'une cassure car le risque d'accidents et d'impacts est plus important pour la } \\
\text { circulation locale. Il s'agit donc d'un ancrage factuel car l'opérateur connaît } \\
\text { l'existence de la communauté même s'il ne peut pas la voir. }\end{array}$ \\
\hline $\begin{array}{l}\text { Fond du tronçon du canal } \\
\text { Enterré X suspendu } \\
\text { (pontillon) }\end{array}$ & $\begin{array}{l}\text { Les tronçons suspendus, ce que l'on appelle "ponts suspendus", possèdent une } \\
\text { structure plus résistante car leur base est en acier et ciment. Les tronçons } \\
\text { enterrés, dont la base n'est pas visible, possèdent des structures moins } \\
\text { résistantes et sont donc plus propices aux cassures et fuites. Il s'agit donc } \\
\text { d'un ancrage réel car l'opérateur voit de quelle structure il s'agit en regardant } \\
\text { directement le canal. }\end{array}$ \\
\hline
\end{tabular}

En ce qui concerne les "évènements historiques ", troisième point contribuant au "jugement de criticité de fuite ou rupture», tous les ancrages sont factuels et font référence aux points qui ont déjà présenté des cassures ou des fuites, aux aspects du canal et aux incidents qui ont impacté sa structure; donc à sa résistance actuelle. Ces faits ont des impacts sur la nature et la criticité des problèmes identifiés sur le moment et requièrent plus d'attention de l'opérateur de terrain durant l'inspection pour les points qui ont déjà fait l'objet d'incidents par le passé.

finalement quant à la question du "contexte ", si c'est l'hiver, il existe de grandes différences de températures entre le jour et la nuit entraînant des chocs thermiques qui aggravent la situation d'éventuelles fissures. En outre, l'évaluation du «poids de l'eau ", qui traduit le sentiment de la quantité d'eau présente dans le canal ou à venir, peut empirer ou atténuer ce jugement de criticité. Il faut souligner que le jugement perceptif composé du " poids de l'eau » est de niveau 2, ce qui signifie qu'il existe au moins un jugement de niveau 1 situé en contrebas ainsi que des ancrages et/ou jugements perceptuels simples. Ces éléments n'ont pas été inclus dans la Figure 4 pour une question d'espace, mais sont présents dans la Figure 5.

Le résultat du "jugement de criticité de rupture ou fuite» fait que l'opérateur doit réaliser une des actions de la Figure 4, représentées par les flèches vertes «plus larges ». Nous avons observé trois actions principales :

- Criticité importante : demande une action urgente pour retirer immédiatement l'eau du canal ;

- Criticité moyenne : d'autres opérateurs sont consultés avant de décider ce qui sera fait ;

- Criticité faible : de moindre urgence, elle indique qu'il faut accompagner l'évolution des signaux au fur et à mesure des jours. 
Pour éviter de nous répéter, nous ne ferons pas de description détaillée de tous les ancrages et actions de l'Arbre. Il est important d'observer que tous les points ont été identifiés en accompagnant les inspections auprès des opérateurs. Nous avons pris en photo pour les autoconfrontations postérieures ce qu'ils considéraient important ; ce qui nous a permis d'identifier les points d'attention pour accélérer l'apprentissage des novices. Cependant, ce n'est que l'expérience des différentes situations en pratique qui permettra de fait aux apprentis de développer leurs jugements pour évaluer la criticité d'une fuite. Lorsque les opérateurs locaux ont eu accès aux arbres, ils ont tout de suite reconnu leur utilité et leurs limites :

«On sait tant de choses que l'on ne le savait pas, n'est-ce pas?»/ « Ah si j'avais pu travailler comme ça quand je suis arrivé ici, j’aurais pu apprendre plus vite!» /

«Très bien ce travail, mais ça va prendre encore un certain temps avant que les novices apprennent tout ça ici. » (Opérateur de terrain)

\section{Arbres séquentiels}

Les arbres séquentiels ont le même objectif que ceux de jugement. Ils sont néanmoins différents, car ils présentent la séquence des actions à réaliser dans le cadre d'une activité. Il existe donc un élément supplémentaire qui est le "contexte ». Cet élément doit être évalué avant l'action pour éviter les risques. Cet arbre ajoute de la valeur à la façon dont les procédés traditionnels tentent de formaliser l'activité humaine, car ils présentent des aspects du contexte. Les jugements et ancrages organisés placés en amont de chaque action se transforment alors en séquences-types, comme c'est le cas du lancement de l'Unité Génératrice (UG) présentée dans la Figure 5. 
Figure 5 : Extrait de l'Arbre séquentiel du lancement de l'unité génératrice (UG). Figure 5: Extract from the Sequential Generating Unit (UG) Starting Tree

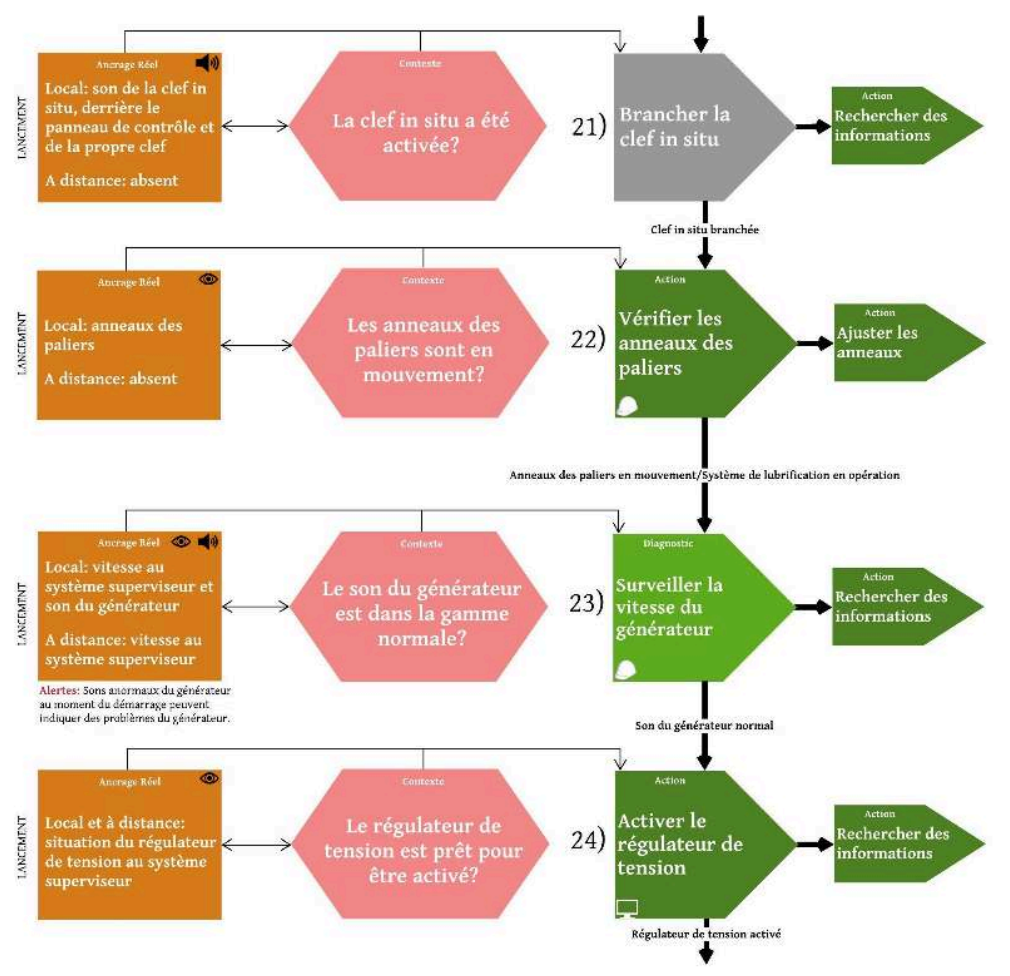

Source : Situated Consultoria e Pesquisa d'apprentissage aux opérateurs expérimentés. Une grande partie de leur travail réalisé de façon tacite ou non-consciente finit par apparaitre dans les arbres, dans les discussions conjointes ou lorsque les opérateurs expérimentés les expliquent aux novices. Il y a donc un échange inespéré d'expériences entre eux, qui est allé au-delà de la demande initiale de l'entreprise et qui a renforcé la contribution que cette formalisation, bien que limitée, peut apporter à la formation de tous les opérateurs.

Pour avoir une idée de l'extension et de la profondeur de l'analyse réalisée durant les 10 mois de durée de l'étude, nous avons analysé 19 activités dans le cadre desquelles 
nous avons identifié 99 jugements tacites, dont certains étaient présents dans plus de 6 arbres des causes. 13 arbres séquentiels ont été créés. Pour les jugements tacites, 41 d'entre eux étaient des jugements perceptuels simples et 58 étaient composés (12 anticipatoires) allant jusqu'au niveau 5. En contrebas, 347 ancrages ont été identifiés dont 262 ancrages réels et 16 ancrages vécus.

\subsection{Conception : modèle analytique pour l'automation anthropocentrique et appropriable}

54 Un autre produit développé est celui d'un modèle analytique pour viabiliser un projet d'automation qui soit appropriable dans le futur par les opérateurs et dont le processus de conception est basé sur les actions de ces derniers. Ce modèle nous a demandé de vérifier si les opérateurs à distance possédaient (ou non) les mêmes ancrages disponibles que ceux des opérations locales. Cette analyse a abouti sur un ensemble de recommandations basées sur le «transfert des ancrages" identifiés durant les opérations locales pour servir de support aux opérations à distance. Nous avons ainsi garanti: (1) que les opérateurs à distance disposeraient de tous les ancrages pour réaliser les jugements nécessaires; (2) d'éviter les «boites noires » dans l'automation des sites et dans le système informatisé de contrôle (système de supervision) qui auraient compliqué les ajustements encore à réaliser par les opérateurs de terrain. Une des situations analysées était le réglage de l'énergie générée.

«L'ajustement fin» consiste à augmenter ou à diminuer la puissance des Unités Génératrices en modifiant leurs charges. À partir de la programmation de la génération, repassée à l'opérateur local par l'équipe de programmation hydroénergétique, l'ajustement fin permet de respecter très minutieusement l'objectif communiqué dans les limites opérationnelles de l'usine du moment et de façon à maintenir la stabilité de l'opération à long terme - c'est-à-dire, pour faire une modification qui n'exige pas d'autre ajustement à court terme.

$\mathrm{Vu}$ de l'extérieur, le comportement observable de l'opérateur consiste seulement à ouvrir ou fermer manuellement une vanne de l'UG dans la PCH (Figure 6). Néanmoins, pour réaliser cette action, l'opérateur prend divers facteurs en considération: (i) l'économie et la meilleure utilisation de l'eau en fournissant la quantité d'eau disponible pour la génération à un moment précis et selon la programmation communiquée (qui tient compte de la génération journalière, mensuelle et annuelle) ; (ii) l'utilisation optimale de l'équipement, c'est-à-dire comment profiter de chaque unité génératrice pour chaque usine en tenant compte des particularités, limites et performances; (iii) la protection des actifs de l'entreprise en préservant les machines et la sécurité des communautés avoisinantes en évitant la rupture des canaux. 
Figure 6 : L'ajustement fin en tant que « comportement observable ». Figure 6: Fine Tuning as "Observable Behavior"

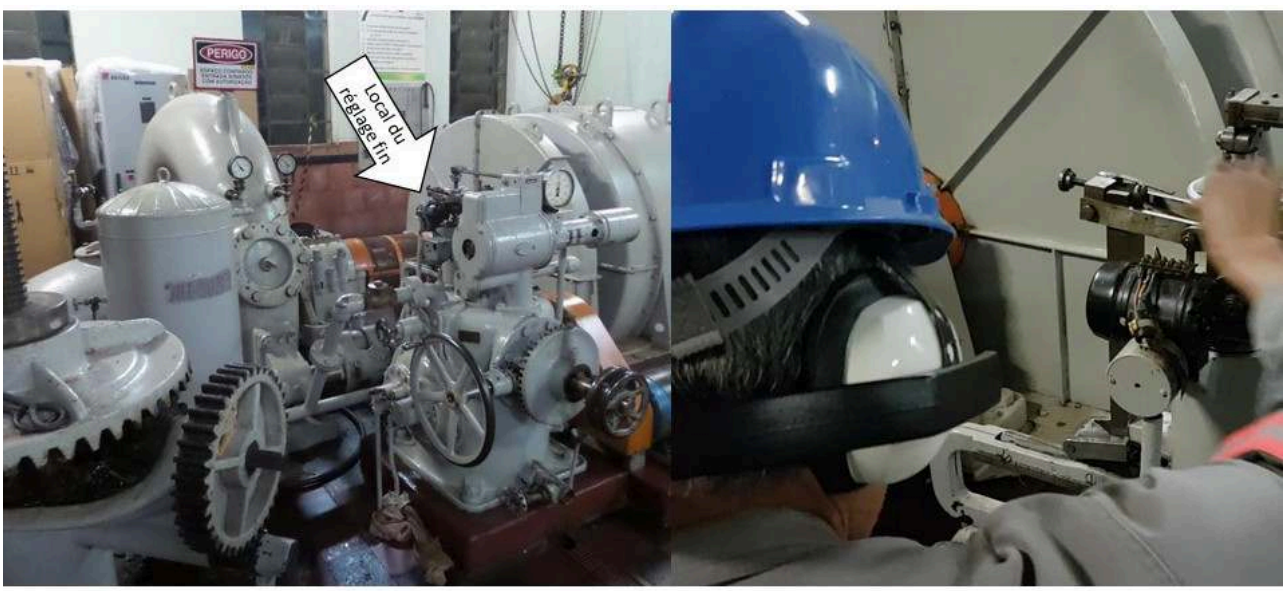

Source : Situated Consultoria e Pesquisa

Par les quatre rectangles gris, l'arbre indique dans quelles zones se trouvent les jugements et ancrages pour réaliser cette action (Figure 7) : barrage, canal, usine ou sous-station. Outre la localisation, les jugements et ancrages sont représentés selon leur temporalité (flèche noire en haut de l'arbre), ou s'ils sont en lien avec l'historique de l'opérateur (son expérience), au passé récent (quelques semaines avant), au présent et au futur (projections/anticipations que l'opérateur fait pour cette action).

Figure 7 : Indication de la localisation et la temporalité des ancrages et jugements. Figure 7: Indication of the Location and Temporality of Anchors and Judgments

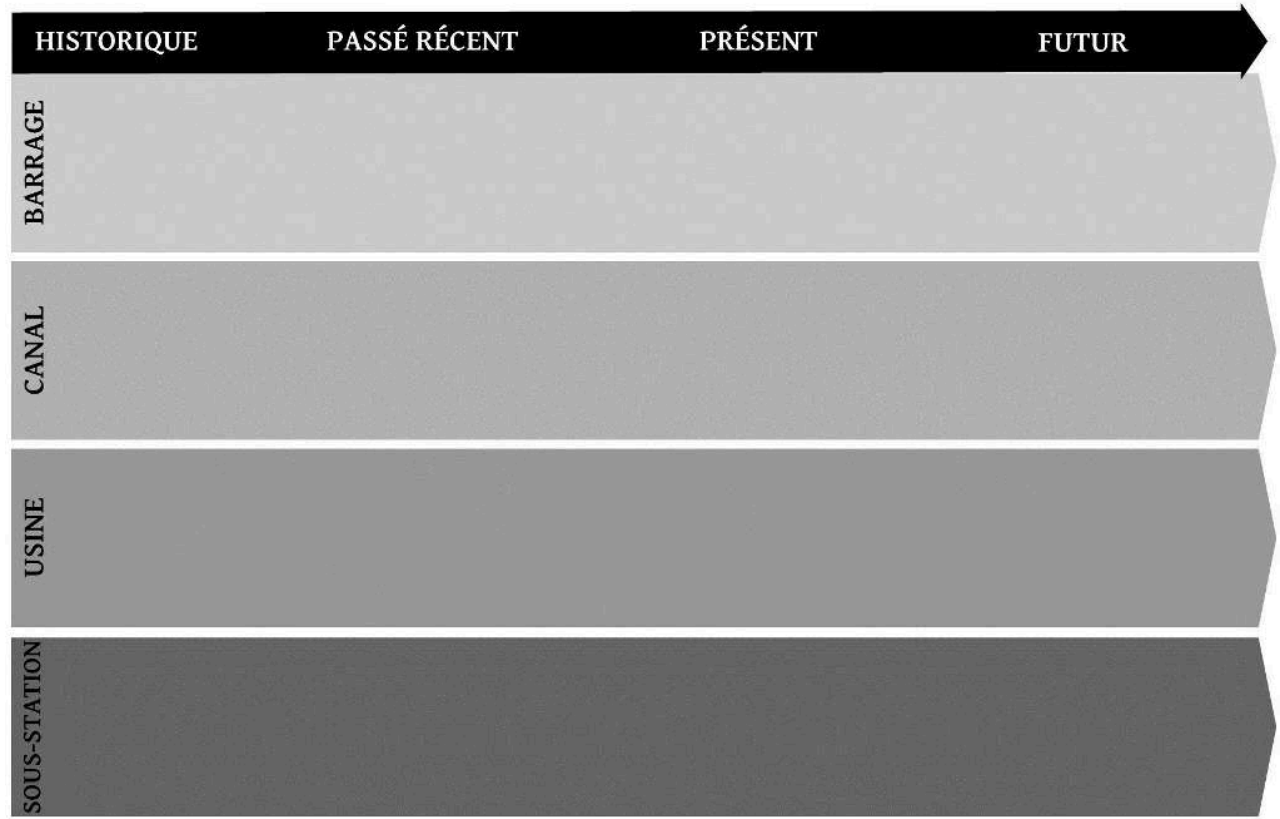

Pour effectuer l'action d'ajustement fin (en vert), l'opérateur local dans l'usiner fait un jugement anticipatoire (en bleu) du temps de réaction et de stabilité de la génération (Figure 8). Il réalise donc deux anticipations: (i) du temps nécessaire de réponse à l'ajustement pour la situation en question; (ii) du temps de stabilisation de 
l'ajustement, c'est-à-dire, pour combien de temps cet ajustement sera suffisant pour garantir la génération sans avoir besoin de faire un autre ajustement à court terme.

Figure 8 : Jugement anticipatoire du temps de réaction et de stabilité de la génération. Figure 8: Anticipatory judgment of reaction time and generation stability

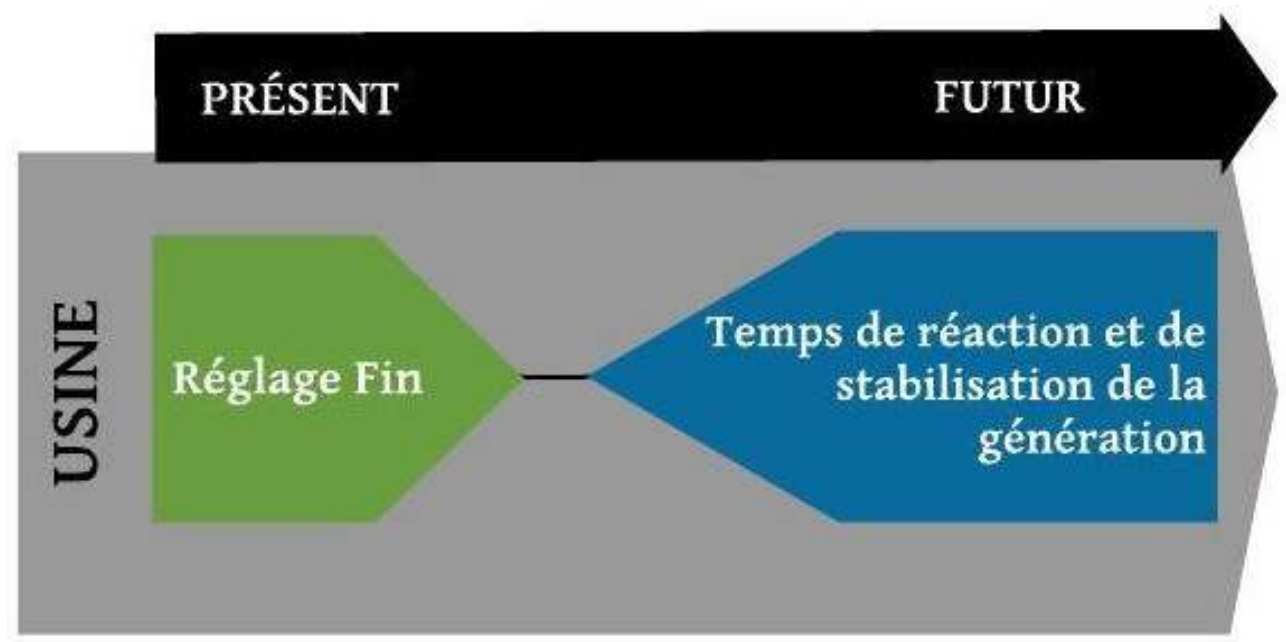

Ce jugement anticipatoire est très complexe et en lien avec une anticipation (futur) qui, pour être réalisée, requiert deux jugements composés au moment de l'ajustement (présent). Il s'agit de «sentir le canal» et de «dominer la machine». La Figure 9 montre l'Arbre des causes de l'ajustement fin pour lequel il est important de visualiser comment les ancrages et les jugements sont distribués sur la temporalité du cours de l'action de l'ajustement de la génération. L'expérience de l'opérateur est identifiée dans les différents endroits où il voit et perçoit les ancrages, dans le passé ou dans le présent. L'arbre démontre une action réalisée dans le présent, le passé y figure de manière dynamique grâce à l'instance de référentiel appelée ici « ancrage factuel » et « ancrage vécu ». 
Figure 9 : Arbre des causes «Ajustement fin » : le comportement du point de vue de l'opérateur. Figure 9: "Fine Adjustment" Tree: the behavior from the operator's point of view

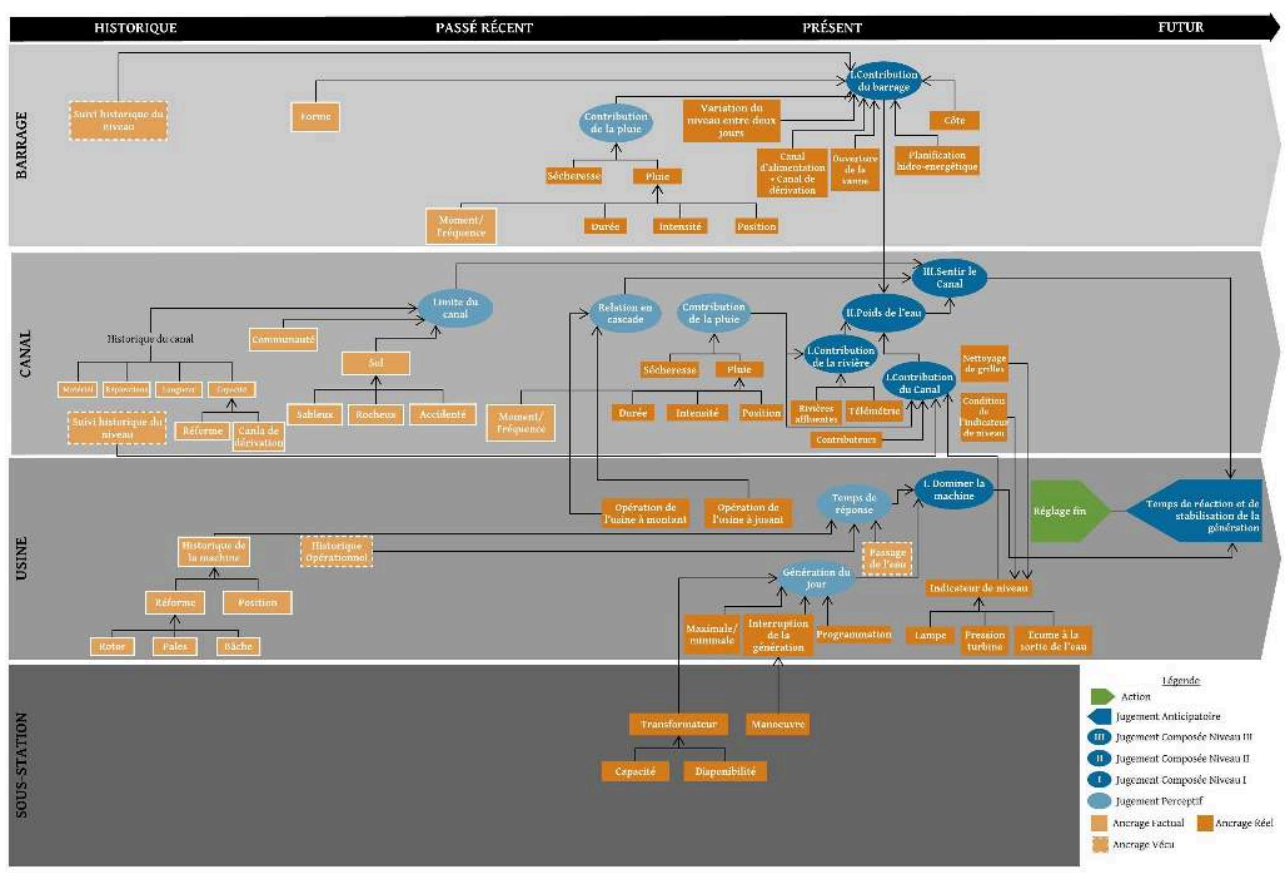

Source : Situated Consultoria e Pesquisa

$60 \mathrm{Au}$ début de l'opération à distance, il est commun que les opérateurs en salle de contrôle effectuent des "ajustements grossiers" et l'opérateur local s'en rendait compte tout de suite et évaluait dans combien de temps il serait nécessaire de changer à nouveau la puissance du générateur. Cette situation montre comment une activité qui, du point de vue de l'observateur externe, serait un comportement observable « simple » (Figure 6) alors qu'en réalité, elle est complexe et imprégnée par différentes temporalités d'action, lorsqu'elle est analysée du point de vue de l'opérateur (Figure 9): (i) le passé grâce à une expérience vécue démontrant ponctuellement ce qu'il a vécu et qui est important pour cette action; (ii) le présent qui montre ce qu'il perçoit au moment d'agir ; et (iii) le futur avec les anticipations nécessaires pour être en accord avec « où l'on veut arriver" .

61 Après avoir effectué la construction et la systématisation des ancrages et leurs localisations, nous avons analysé quels sont les ancrages réels perçus par l'opérateur local (visuels, auditifs, olfactifs, etc.) pour chaque action et qui, à leur tour, lui permettent de développer des «backgrounds vécus » incorporés (ancrages vécus) et la possibilité de réaliser des jugements simples et composés selon la situation. Cette analyse permet d'observer comment ces ancrages sont disponibles pour les différentes équipes (locale et à distance). Lorsqu'ils sont présents pour la supervision, cela veut dire qu'ils sont accessibles aux opérateurs à distance. Mais quand ils sont uniquement " très locaux ", c'est-à-dire dans l'équipement même ou dans les zones des usines, nous considérons qu'ils sont inaccessibles à distance.

62 Cette analyse est importante pour vérifier les actions qui peuvent être réalisées par chaque équipe (locale et à distance). Dans le but d'augmenter l'efficacité de la performance de l'activité, la sécurité et la protection des actifs du complexe énergétique, nous suggérons que l'activité ne soit réalisée par une équipe déterminée que lorsque tous les ancrages sont disponibles pour exécuter les actions. Nous avons 
ainsi créé une classification des ancrages après l'automation avec le type d'opération demandée dans chaque cas: uniquement local, uniquement à distance ou en coopération (opération locale + à distance). Le modèle analytique a été élaboré pour servir de base technique à la mise en place de la co-opération et a contribué à l'élaboration ou au perfectionnement de la Matrice des Responsabilités pour chaque activité et pour les décisions collectives. En outre, ce modèle a également permis d'affiner l'automation déjà effectuée et de guider les futurs projets à réaliser dans les usines en cascade.

Un système automatisé peut englober, comme une partie de ses algorithmes, des règles de désarmement ou d'alarme, par exemple, dans le cas où le processus atteint une certaine température ou lorsqu'un niveau de matériel dans un réservoir arrive à une certaine hauteur. L'aptitude de réaliser des jugements anticipatoires des opérateurs de $\mathrm{PCHs}$ est donc une partie importante de leur activité et elle n'est possible que si la perception humaine "perçoit " également le sens de la situation. La perception du risque et de l'urgence, par exemple, n'est ressentie que par ceux qui sont capables d'anticiper les conséquences en cas d'incident. L'automation, alliée aux standards et procédés opérationnels, essaie de systématiser les aspects de l'expérience humaine, mais elle en sera toujours dépendante. Cela ne signifie pas que l'automation ne puisse pas remplacer certains opérateurs de terrain par un opérateur à distance ou que l'automation ne fonctionne pas. Ces cas nous montrent seulement que le savoir tacite ne peut être complètement formalisé et ce qui est produit avec la "formalisation" (procédures, règles, algorithmes, automatismes, etc.) dépend du savoir tacite des professionnels expérimentés afin de pouvoir fonctionner correctement ou être bien utilisé. Le Modèle Analytique créé analyse chaque ancrage d'une activité versus le système automatisé et les classe comme suit : maintenus, non disponibles, intégrés et remplacés (par le système), comme démontré dans la Figure 10. Nous présentons cidessous chaque catégorie. 
Figure 10 : Modèle Analytique pour l'Automation Anthropocentrée et Appropriable. Figure 10: Analytical Model for Anthropocentric and Appropriable Automation

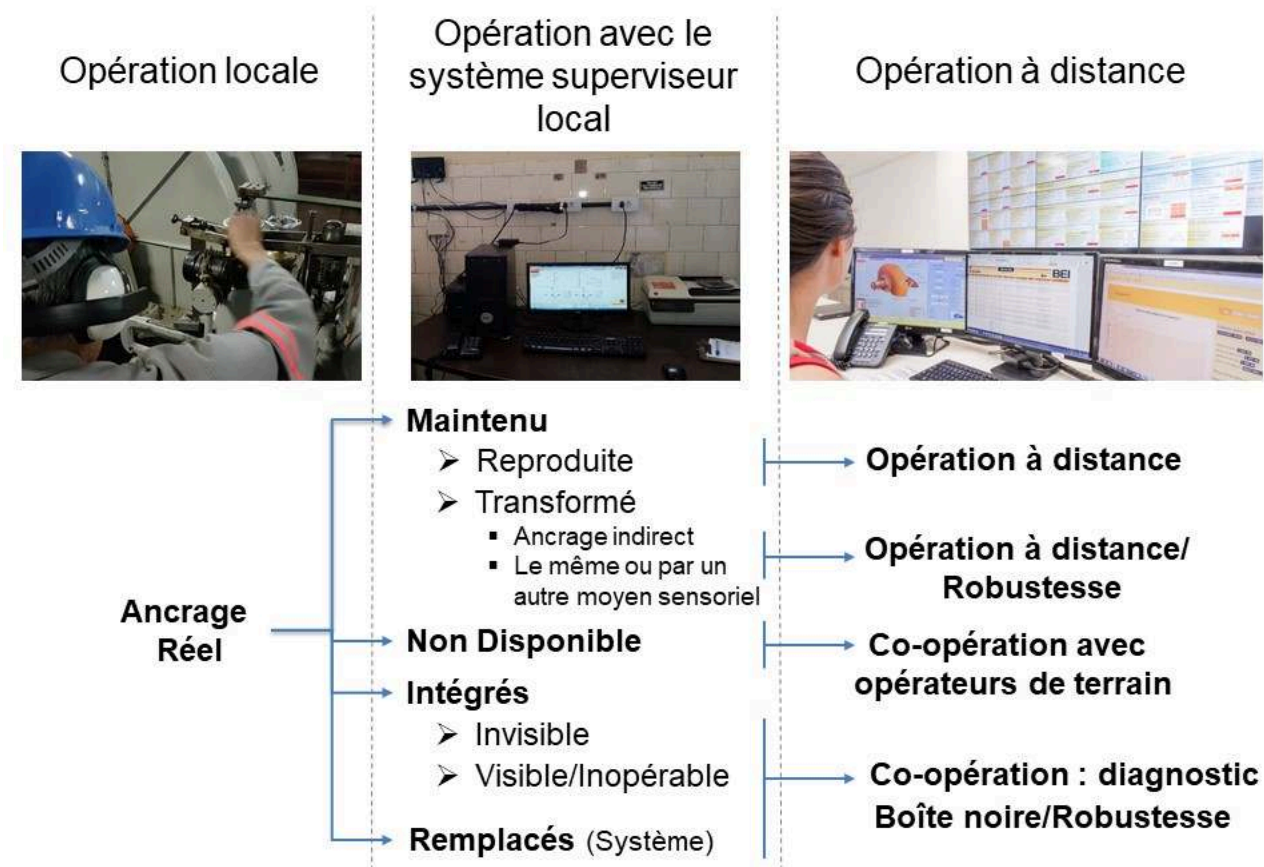

Source : Situated Consultoria e Pesquisa

\subsubsection{Ancrages maintenus}

Lorsque les ancrages réels ressentis par l'opérateur local pour exécuter une action sont disponibles pour l'opérateur à distance, soit grâce au système informatisé de contrôle (ou système de supervision) ou à des caméras, nous considérons qu'ils sont MAINTENUS après l'automation. Il est ainsi possible de réunir les conditions pour que l'opérateur à distance développe les facultés nécessaires pour donner du sens à ce qu'il ressent dans le cadre des différents contextes. Ces ancrages peuvent être maintenus de deux manières :

- REPRODUITS : lorsque les ancrages sont accessibles de la même façon aux opérateurs locaux et à distance, nous disons qu'ils ont été maintenus et reproduits. À titre d'exemple, c'est le cas lorsqu'une caméra est placée dans la gouttière et permet de visualiser l'écoulement de l'eau à un moment précis.

- TRANFORMÉS : lorsque les ancrages sont accessibles à l'opérateur à distance, mais qu'ils ont été traduits pour être à disposition de la supervision, nous disons qu'ils ont été maintenus ou transformés. Cette transformation peut avoir lieu grâce à un ancrage "indirect » qui utilise le même ou un autre moyen sensoriel. Comme exemple de moyen sensoriel : vérifier si le disjoncteur est armé ou désarmé. Cette action était réalisée visuellement avec le propre équipement et elle est toujours encore faite visuellement, mais à partir de l'écran du système de supervision. Exemple de modification sensorielle : vérifier la vitesse de l'UG. La vitesse était estimée par le bruit du générateur et a été transformée en une mensuration des rotations par minute (rpm). 
Pour les deux formes d'ancrages maintenus, l'action peut être réalisée à distance ; la coopération n'est nécessaire que dans les cas de diagnostic correctif et pour vérifier si la transformation réalisée est réellement une opération à distance solide, c'est-à-dire si les données reproduites et transformées sont fiables ou s'il est nécessaire d'introduire une redondance pour valider un indicateur.

\subsubsection{Ancrages non disponibilisés}

Lorsque les ancrages nécessaires pour prendre une décision et réaliser une action ne sont disponibles que pour l'opération locale, c'est-à-dire quand ils n'ont pas été insérés dans le système de supervision ou qu'ils ne sont pas disponibles par vidéo, nous disons que ce sont des ancrages NON DISPONIBILISES pour les opérations à distance. Nous pouvons citer l'exemple de l'interrupteur de déconnexion, pour lequel la supervision ne sait pas dans quelle position réellement il se trouve et son opération ne peut être réalisée que localement. Ainsi, lorsqu'il s'agit d'un ancrage non disponibilisé, l'action ne peut être réalisée que localement pour augmenter la sécurité et la protection des actifs.

\subsubsection{Ancrages intégrés}

67 Avec l'automation, certaines opérations, avant effectuées directement par l'opérateur, ont été transformées en opérations réalisées par le propre système. Elles existent donc encore, par exemple, l'ouverture automatique de la vanne papillon, mais c'est le système qui les contrôle et agit postérieurement dans ce cas. Nous disons donc que ces ancrages sont INTÉGRÉS, car ils étaient déjà présents, mais sont soumis au contrôle fait par le système automatisé. Ces ancrages sont présents sous deux formes :

- INVISIBLES : certains ancrages ne peuvent être perçus après l'automation bien qu'ils soient contrôlés par le système automatisé.

- VISIBLE/INOPÉRABLE : certains ancrages restent disponibles pour les opérateurs après l'automation bien que l'opération soit effectuée par le système. Les opérateurs réussissent donc à les remarquer sur le moment, mais ne peuvent pas agir sur eux. C'est le cas, par exemple, du contrôle des paramètres de fréquence, tension et courant pour opérer manuellement le disjoncteur. Aujourd'hui les mesures de ces trois paramètres sont disponibles pour l'opérateur, mais le disjoncteur est opéré par le système.

Dans les deux formes d'ancrages intégrés, nous suggérons de vérifier la robustesse de l'automation pour contrôler et exécuter les opérations. Si l'ancrage intégré est invisible, cette robustesse est encore plus importante, car le diagnostic est confronté à une "boite noire ". En d'autres termes, l'opérateur n'a pas accès aux données à percevoir et à analyser en cas de panne. Quant à l'ancrage intégré visible/inopérable, le diagnostic correctif est facilité, car l'opérateur est en possession des données/signaux à percevoir et à analyser en cas de panne. Outre le diagnostic correctif, en cas de disponibilité des ancrages pour l'opération à distance, les conditions nécessaires sont présentes pour le développement de la faculté d'anticipation et d'action pour éviter les pannes et incidents. 


\subsubsection{Ancrages remplacés (système)} l'opérateur comme, par exemple, faire la synchronisation ont été complètement exclues ou transformées en opérations dans le système automatisé, mais de façon totalement différente de la procédure originale. Nous disons alors que ces ancrages ont été REMPLACÉS et ne sont plus accessibles à l'opérateur local et à distance. Dans ce cas, il existe une "boite noire» pour ces opérations, car l'opérateur ne dispose plus de données/signaux à remarquer et à analyser durant l'activité. Nous suggérons de vérifier la robustesse de l'automation pour l'exécution de ce genre d'opération.

Pour faciliter la classification des ancrages, nous avons élaboré un tableau (Tableau 4) avec les ancrages d'une activité réalisée par les opérateurs locaux qui montre également les éventuelles conséquences si une activité est réalisée sans que certains ancrages importants pour l'action soient mis à disposition. Ceci nous a conduits à suggérer certaines améliorations du système automatisé afin qu'il soit plus adhérent au travail des opérateurs. À titre d'exemple, nous avons inclus et perfectionné les ancrages pour les opérateurs à distance de façon à transformer certaines «boites noires " du système automatisé en « boites transparentes » (Lave \& Wenger, 2009 [1991], p. 102), ce qui a permis une meilleure compréhension de la logique de certains automatismes et de leur contrôle par les opérateurs. Grâce aux modifications des conditions techniques en quête d'une action plus efficiente et sûre, nous avons trouvé la voie pour la mise en place d'un système plus anthropocentrée et appropriable, car plus adhérent et transparent.

Tableau 4 : Modèle Analytique de Co-opération. Exemple : Démarrage de l'unité de génération Arbre 14.

Table 4: Analytical Co-Operation Model. Example: Start of generating unit - Tree 14

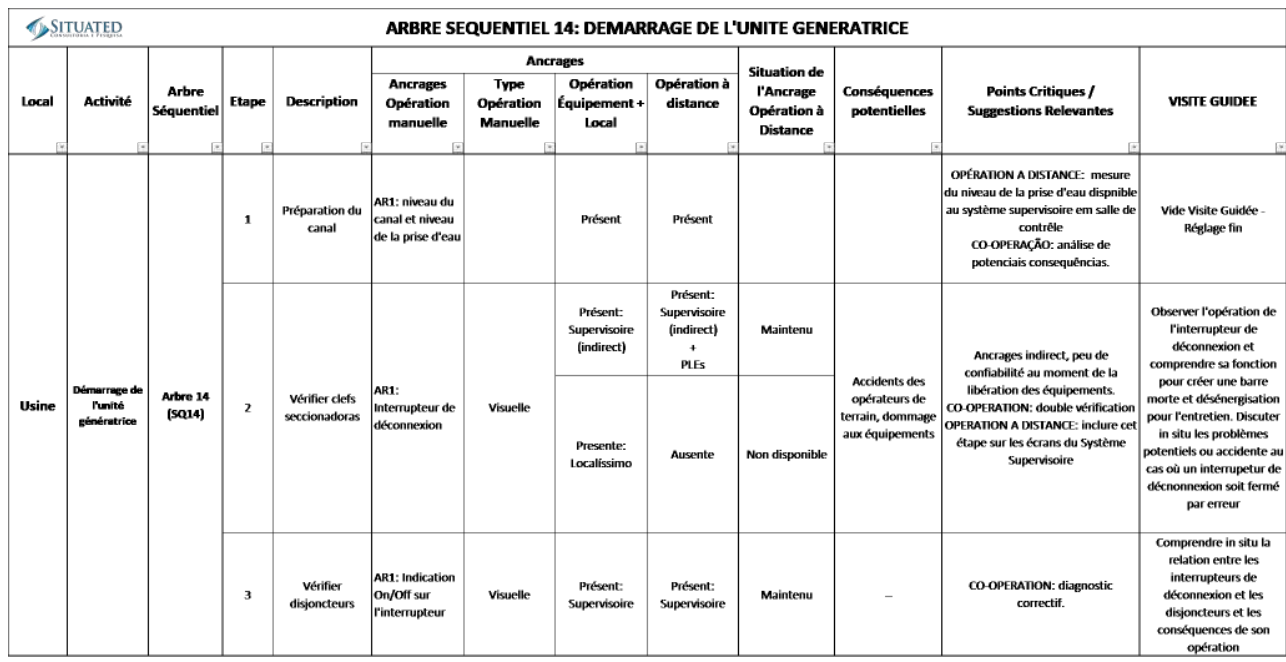

\subsection{Co-opération H-H-M : Modèle Analytique : Opération locale versus Opération à distance}

71 Un troisième ensemble de propositions concerne l'organisation et la division du travail entre les opérateurs locaux et à distance. Il a fallu former les opérateurs à distance, que nous abordons dans cette partie, séparément de la formation des opérateurs de terrain, 
car cette formation était le point de départ pour instituer une nouvelle division des tâches et pour débuter un processus de coopération entre opérateurs de terrain et de la salle de contrôle. Il s'agit à vrai dire d'une co-opération, car certaines décisions et ajustements sont à partager entre les deux catégories et augmentent ainsi la liste des tâches et l'autonomie des opérateurs de terrain. Certaines décisions vont devoir être prises collectivement et d'autres incomberont aux opérateurs de terrain qui peuvent évaluer et mieux décider que faire au bon moment. D'autres opérations seront exclusivement réservées aux opérateurs à distance. Dans la Figure 5, par exemple, il y a des actions dans les arbres séquentiels qui incombent à l'opérateur local (identifiées par un "casque ») ou à l'opérateur à distance (identifiées par un " écran »). Il existe également des actions, non présentes dans cette figure, où apparaissent les deux symboles, pour démontrer la nécessité d'une opération conjointe. C'est ce que nous appelons la co-opération.

Le « Modèle Analytique Opération Locale x à Distance », allié au modèle précédent, a comme objectif principal de fournir des conditions pour analyser l'automation - déjà existante ou future - d'une activité déterminée. En d'autres termes, il s'agit de vérifier si l'automation réalisée (ou à réaliser) est adhérente à l'activité exécutée localement. Il est ainsi possible: (i) de perfectionner les systèmes automatisés existants; (ii) de concevoir des systèmes d'automation plus adhérente (en évitant le retravail postérieur) et (iii) d'identifier les activités pour lesquelles une co-opération est nécessaire entre les opérateurs locaux et à distance, même après la finalisation du processus d'automation. Cette dernière analyse permet également d'élaborer une matrice de responsabilités partagées entre les opérations locales et à distance, de fournir un support au dimensionnement des équipes adapté aux besoins de la génération et simultanément de protéger les infrastructures - un point important dans le quotidien opérationnel des usines en cascade.

Après la définition des Matrices de Responsabilités, avec l'attribution des activités à réaliser par l'équipe locale, à distance ou en co-opération, nous avons organisé des visites guidées dans le complexe des usines pour les opérateurs à distance. Ce sont les opérateurs locaux qui ont participé à l'élaboration des arbres qui ont guidé ces visites dans le but de leur faire approprier en partie les principaux ancrages réels qui leur servent de base pour leurs jugements et actions. Ces visites ont donné lieu à des transformations significatives pour les opérateurs à distance qui ont arrêté de se sentir hiérarchiquement supérieurs par rapport à leurs collègues du terrain. Certaines phrases des opérateurs à distance pendant les visites témoignent du changement opéré : "Notre chef parle, mais je n'avais aucune idée des proportions » / "Maintenant je sais ce qu'ils veulent dire / "Nous, on n'a même pas les paramètres de ces règles! [à propos des règles physiques]». Après la formation des opérateurs à distance, les opérateurs locaux sont moins préoccupés et font plus confiance à leurs collègues à distance : « Maintenant, on leur demande moins d'augmenter la puissance pour diminuer l'eau du canal! »

Sur la base de ces interventions, il n'a pas encore été possible de réaliser la conception des systèmes de supervision et des interfaces des systèmes informatisés de la salle de contrôle. Une description plus détaillée des processus décisionnels comprenant la question de « sentir le canal » et d'autres activités spécifiques des opérateurs de terrain pourraient créer des modèles proposés par la théorie du cours de l'action et orienter les actions des opérateurs à distance ainsi que leur formation. 


\section{Conclusion}

Pour quelle raison assistons-nous encore actuellement, dans le cadre de l'industrie 4.0, à la répétition d'équivoques telles que les usines sans hommes dont les ingénieurs rêvaient depuis les années 1950 ?

Les promesses de la première vague d'Intelligence Artificielle des années 1950 ont été amplement critiquées (Collins, 1992; Dreyfus, 1984; Winograd \& Flores, 1989) et invalidées par l'expérience pratique, mais réapparaissent toujours. Pour ne citer qu'un exemple, dans les années 1990, Dertouzos, un spécialiste du MIT reprend le même leitmotiv tout en reconnaissant, après presque 50 ans de tentatives, que les technologies ont encore besoin d'évoluer : «Les machines automatiques devraient être perfectionnées à fond pour produire des biens et services sans l'aide des hommes " (Dertouzos, 1997, p. 340). Avec la promesse de l'automatisme sans les hommes, l'auteur nous présente un projet de société : «Dans une société libre du travail, les personnes ne seraient plus obligées de travailler pour vivre» (idem p. 341). Cette situation ne s'est pas concrétisée en raison du consumérisme des individus et non pas à cause des relations sociales de production:

«Le temps pour les loisirs en fait a diminué. Bien que notre productivité ait augmenté et que nos besoins de base soient satisfaits, nous préférons travailler encore plus pour avoir un meilleur niveau de vie et acheter des produits de luxe... » (Dertouzos, 1997, p. 341).

« Nous serons tous capitalistes, car nous posséderons tous des machines et d'autres biens de capital générateurs de bénéfices » (Idem, p. 342).

77 Nous retrouvons ici la vision capitaliste néolibérale, celle qu'Aristote avait déjà anticipée avec plus de grandeur :

Si chaque instrument, en effet, pouvait, sur un ordre reçu, ou même deviné, travailler de lui-même, comme les statues de Dédale, ou les trépieds de Vulcain, "qui se rendaient seuls, dit le poète, aux réunions des dieux»; si les navettes tissaient toutes seules; si l'archet jouait tout seul de la cithare, les entrepreneurs se passeraient d'ouvriers et les maîtres d'esclaves. (Aristóteles, 2006, p. 10-11)

78 Ces utopies technologiques conservent leur éternelle jeunesse en raison de la double ingénuité des ingénieurs: (i) le rationalisme technique qui étaye la croyance que l'Intelligence Artificielle est possible et (ii) que la technologie en soi peut remédier aux maux de la société - ces deux croyances sont actuellement renforcées par un terrible déni social à l'égard du travail par l'avancée du courant néolibéral. Nous retrouvons ici la question de l'aspect anthropologiquement constitutif de la technique adoptée de façon bien plus consistante par la théorie du cours de l'action dans sa vocation de transformer la conception ergonomique en un instrument de transformation sociale. Avec nos exemples relativement réduits, dans le but d'opérer des transformations locales, nous avons montré de façon empirique ce que les descriptions de l'activité dans des modèles du cours de l'action ou des systématisations de ses éléments peuvent promouvoir en termes de modifications techniques et de changement des relations sociales de façon intégrée.

Le projet de départ de l'entreprise était de créer des PHCs non-habitées, opérées seulement à distance par des techniciens formés seulement pour contrôler des systèmes informatisés. Finalement, nous avons pu montrer que ce modèle ne pouvait pas fonctionner et un système co-opératif a été adopté avec des décisions prises de forme collaborative par les opérateurs à distance et de terrain. Quant à la présence de 
ces derniers, lorsque l'automation acquiert une stabilité relative, elle permet de réduire la quantité de ces opérateurs qui ne se consacrent plus à une seule usine et dont la polyvalence doit être développée plus rigoureusement avec l'aide des arbres des causes. Ces derniers contribuent ainsi, non pas à éliminer les opérateurs de terrain, mais à faciliter le développement du savoir tacite nécessaire aux jugements situés, sur le terrain comme à distance. A notre avis, ceci peut être une démonstration palpable d'un aspect de que peut représenter « une humanité désirée » (Theureau, 2004).

\section{BIBLIOGRAPHIE}

Aristóteles. (2006). A Política. São Paulo : Martins Fontes.

Assunção, A. A. (1998). De la déficience à la gestion collective du travail. Thèse de Doctorat d'Ergonomie. Paris, EPHE.

Bijker, W. E., Hugues, T.P., \& Pinch, T. (1989). The social construction of technological systems. Cambridge : MIT Press.

Böhle, F., \& Milkau, B. (1988). De la manivelle à l'écran. L'évolution de l'expérience sensible des ouvriers lors des changements technologiques. Paris : Eyrolles.

Brödner, P. (1990). Technocentric-Anthropocentric Approaches. In M. Warner, W. Wobbe, \& P. Brödner (Eds.), New technology and manufacturing management. (pp. 101-111). New York : John Wiley.

Brödner, P. (1993). Anthropocentric production systems. In G. Salvendy, \& M. Smith (Eds.), Human-computer interaction. (pp. 74-79). Amsterdam : Elsevier.

Caroly, S. (2012). Gestion collective de situations critiques au guichet en fonction de l'âge. In A. F. Molinié, C. Gaudart, \& V. Pueyo (Eds.), La vie professionnelle : âge, expérience et santé à l'épreuve des conditions de travail (pp. 223-234). Toulouse : Octarès.

Clot, Y. (2010). Trabalho e poder de agir. Belo Horizonte : Fabrefactum.

Collins, H. M. (1992). Experts artificiels. Paris : Seuil.

Corbett, J. M., Rasmussen, L. B., \& Rauner, F. (1991). Crossing the border: the social and engineering design of computer integrated manufacturing systems. Berlin : Springer-Verlag.

Daniellou, F. (2007). A ergonomia na condução de projetos de concepção de sistemas de trabalho. In P. Falzon (Ed.), Ergonomia (pp. 303-316). São Paulo : Edgard Blucher.

Dertouzos, M. (1997). O que será? São Paulo : Companhia das Letras.

Dreyfus, H. (1984). Intelligence Artificielle : mythes et limites. Flammarion.

Duarte, F. M. C., \& Lima, F. P. A. (2012). Anticiper l'activité par les configurations d'usage. Activités, 9, 22-47. https://doi.org/10.4000/activites.314

Ehn, P. (1988). Work-oriented design of computer artifacts. Stockholm : Arbetslivscentrum.

Eijnatten, F. M. (1993). The Paradigm That Changed the Work Place. Assen : Van Gorcum. 
Engeström, Y. (1987). Learning by expanding. Helsinki : Orienta-Konsultit.

Falzon, P. (2007). Ergonomia. São Paulo : Edgard Blucher.

Falzon, P. (2013). Ergonomie constructive. Paris : PUF.

Ferreira, L. L. (2015). Análises do trabalho. Belo Horizonte : Fabrefactum.

Fernandez, G. \& Clot, Y. (2007). Entrevistas en auto-confrontación: un método en clínica de la actividad. Laboreal, 2(1), 15-19. http://laboreal.up.pt/revista/artigo.php?

id=37t45nSU5471122987296762231

Fernandez, G. (2004). Développement d'un geste technique. Histoire du freinage en Gare du Nord. Thèse de doctorat en psychologie. Paris : CNAM.

Folcher, V., \& Rabardel, P. (2007). Homens, artefatos, atividades: perspectiva instrumental. In P. Falzon (Ed.), Ergonomia (pp. 207-222). São Paulo : Companhia das Letras.

Garrigou, A., Daniellou, F., Carballeda, G., \& Ruaud, S. (1995). Activity analysis in participatory design and analysis of participatory design activity. International Journal of Industrial Ergonomics, 15, 311-327. https://doi.org/10.1016/0169-8141(94)00079-I

Ingold, T. (2001). From the transmission of representations to the education of attention. In H. Whitehouse (Ed.), The Debated Mind: Evolutionary psychology versus ethnography. (pp. 113-153). Oxford: Berg.

Jeffroy, F. (1987). Maîtrise de l'exploitation d'un système micro-informatique par des utilisateurs noninformaticiens. Thèse de doctorat. Paris, Université Paris XIII.

Lave, J., \& Wenger, E. (1991). Situated Learning. Cambridge : Cambridge University Press.

Lima, F. P. A., \& Duarte, F. M. C. (2014). Integrando a ergonomia ao projeto de engenharia: especificações ergonômicas e configurações de uso. Gestão \& Produção. 21, 679-690. http:// dx.doi.org/10.1590/0104-530X733-13

Lima, F. P. A., Duarte, F. M. C., Resende, A. E., Garrigou, A., \& Carballeda, G. (2015). Où est la maîtrise du projet dans les processus de conception dans les organisations complexes. In Actes du $50^{e}$ Congrès de la SELF. Paris : SELF, (Vol. 1). 468-475.

Mackenzie, D., \& Wajcman, J. (Eds.). (1999). The Social Shaping of Technology. Buckingham : Open University Press.

Nardi, B. A. (Ed.) (1997). Context and consciousness. Cambridge : MIT Press.

Pinsky, L. (1992). Concevoir pour l'action et la communication. Berne : Peter Lang.

Pinsky, L., \& Theureau, J. (1987). L'étude du cours d'action. Paris : Ed. CNAM.

Ribeiro, R. (2013). Remarks on Explicit Knowledge and Expertise Acquisition. Phenomenology and Cognitive Sciences, 12, 431-435. https://doi.org/10.1007/s11097-012-9268-9

Ribeiro, R. (2014). The Role of Experience in Perception. Human Studies, 37, 559-581. https:// doi.org/10.1007/s10746-014-9318-0

Rocha, R. (2015). Do Silêncio organizacional aos espaços de debate sobre o trabalho. In F. Lima, L. Rabelo, \& M. Castro (Eds.), Conectando Saberes (pp. 111-140). Belo Horizonte : Fabrefactum.

Rocha, R., Daniellou, F., \& Mollo, V. (2014). O retorno de experiência e o lugar dos espaços de discussão sobre o trabalho: uma construção possível e eficaz. Trabalho \& Educação, 23(1), 61-74. 
Rocha, R., Mollo, V., \& Daniellou, F. (2015). Work debate spaces: A tool for developing a participatory safety management. Applied Ergonomics, 46(a), 107-114. https://doi.org/10.1016/ j.apergo.2014.07.012

Simonet, P. (2009). L'examen méthodique d'un geste de métier pour une prévention durable des TMS. PISTES. http://pistes.revues.org/2404

Stiegler, B. (2018). La technique et le temps. Paris : Fayard.

Theureau, J. (1992/2004). Le Cours d'Action : Méthode Élémentaire. Toulouse : Octarès.

Theureau, J. (2004). L'hypothèse de la cognition (ou action) située et la tradition d'analyse du travail de l'ergonomie de langue française. Activités, 1(2), 11-25. https://doi.org/10.4000/activites. 1219

Theureau, J. (2006). Le Cours d'Action : Méthode développée. Toulouse : Octarès.

Theureau, J. (2009). Le Cours d'Action : Méthode réfléchie. Toulouse : Octarès.

Theureau, J. (2014). o curso da ação: método elementar. Ensaio de antropologia enativa e ergonomia de concepção. Belo Horizonte : Editora Fabrefactum.

Theureau, J. (2015). Le Cours d'Action : L'énaction et l'expérience. Toulouse : Octarès.

Theureau, J. (2019). Le Cours d'Action : Économies et activités. Toulouse : Octarès.

Theureau, J., \& Jeffroy, F. (Eds.). (1994). Ergonomie des situations informatisées. Toulouse : Octarès.

Trinquet, P. (2010). Trabalho e educação: o método ergológico. Revista HISTEDBR On-line, número especial, 93-113.

Vinck, D. (2013). Engenheiros no cotidiano. Belo Horizonte : Fabrefactum.

Winograd, T., \& Flores, F. (1989). L'intelligence artificielle en question. Paris : PUF.

Wisner, A. (2004). Questões epistemológicas em Ergonomia e em análise do trabalho. In

F. Daniellou (Ed.), A ergonomia em busca de seus princípios debates epistemológicos. (pp. 29-56). São

Paulo : Edgard Blucher.

\section{NOTES}

1. Pour une vision générale de ces approches de projet en ergonomie, nous renvoyons le lecteur à l'ouvrage collectif organisé par Falzon (2007). Chez Rabardel, le pôle technologique est central, mais la théorisation s'est arrêtée à la notion de schémas opératoires, inspirée de Piaget, mais qui ne progresse pas dans la modélisation dynamique pour doter la technique de caractéristiques d'appropriabilité, comme souligné dans la TCA (voir partie 3).

2. Cette «explicitation» du tacite est toujours partielle car toute connaissance tacite une fois découverte est « réifiée » (Ribeiro, 2013, p. 431) et exige à nouveau de l'expérience et des habilités tacites pour son utilisation située. Les objets intermédiaires de la formation, les arbres des causes et séquentiels, dans le cas présent, servent à systématiser les "points d'attention " pour les travailleurs expérimentés et les novices en contribuant aux interactions déjà citées.

3. Corbett, Rasmussen et Rauner (1991, p. 13) concluent le rapport des projets avec des notes pessimistes : « Sur la base d'une connaissance intime de l'entreprise pour laquelle il travaille, l'un des ingénieurs participant à notre projet, a affirmé qu'un “autre grand problème est faire en sorte que la gestion traditionnelle accepte que les individus ont besoin d'un niveau élevé de liberté afin d'être flexibles". Notre expérience d'impliquer la gestion des entreprises innovatrices vers un niveau plus élevé de CIM centrés sur l'humain confirme en général que la gestion basée 
sur le marketing, en raison de son affinité intellectuelle avec l'économie appliquée, est plus encline à empêcher toute innovation à long terme que ses paires orientés par l'ingénierie et la production » (Traduction libre)

4. Les auteurs font la différence entre «l'activité objectivante » et «l'activité subjectivant»: «Pour le problème qui nous intéresse ici, il est logique de désigner une activité rationnelle rationnelle selon les critères dominants- pour l'expression d'activité objectivante. On exprime ainsi une caractéristique fondamentale: une telle activité se base sur des connaissances objectives et par conséquent sur des règles universellement reconnues et généralisables " (Böhle \& Milkau, 1988, p. 17). « Avec le concept d'activité subjectivante, nous abordons des formes de perception sensobe qui ne sont pas prises en considération dans les concepts scientifiques dominants, ou qui ne le sont que de façon marginale» (p. 20). "L'activité subjectivante ne vise pas d'abord à acquérir voire à se baser sur des connaissances objectivables sur l'environnement, ni sur les règles d'action correspondantes. Au premier plan, se dressent plutôt, dans le commerce avec les personnes comme avec les choses, justement leurs particularités et leurs variations concrètes, différentes et- en ce sens- uniques. » (Idem, p. 22).

5. Certaines questions sont encore ouvertes pour opérer la transformation entre la description de l'activité, soit sous formes des cours de l'action, et les contenus de ces modèles. La notion de " configuration d'usage" (Duarte \& Lima, 2012; Lima \& Duarte, 2014) cherche à avancer dans cette direction.

6. Cette formalisation basée sur le cours de l'action est orientée, selon les auteurs, vers les concepts d'analyse des situations de référence, de situations d'actions caractéristiques (SACs) et de l'analyse de l'activité future (AAF), proposées par François Daniellou et Alain Garrigou (Garrigou, Daniellou, Carballeda, \& Ruaud, 1995). Néanmoins, dans le cours de l'action, ce sont plutôt des conceptions et des modèles de description de l'activité qui sont développés alors que l'approche de l'AAF s'est penchée plus sur la construction sociale de l'intervention en situation de projet.

\section{RÉSUMÉS}

Depuis la décennie de 1950, les échecs de développement de technologies anthropocentriques laissent à penser que les principes généraux de conception ouvrent la voie aux projets sociotechniques bien que ces derniers soient peu opérationnels pour articuler les aspects sociaux, subjectifs et techniques. Cet article démontre comment la théorie du "cours de l'action », qui appartient au champ de l'ergonomie de "l'activité », contribue à rendre cette articulation effective. Sur la base d'un cas d'automation de petites centrales hydro-électriques (PCHs), où des concepts et méthodes de la théorie du cours de l'action ont été utilisés, nous démontrons la nécessité d'une praxéologie empirique afin de traiter les interfaces entre $\mathrm{H}-\mathrm{H}$ (formation) et $\mathrm{H}-\mathrm{M}$ (automation), appelées aussi systèmes H-H-M. Du point de vue théorique, les contributions de cet article s'inscrivent au niveau du pôle technologique du Programme de Recherche du Cours de l'Action et, en termes pratiques, il s'agit d'une alerte afin que l'industrie 4.0 ne reproduise pas le mythe de l'usine « sans hommes».

Since the 1950s, failures in the development of anthropocentric technologies have suggested that general design principles open the way for socio-technical projects. However, the latter do not sufficiently integrate the social, subjective and technical aspects underlying every kind of 
industrial project. This article shows how the "course of action" theory, which is part of the "activity" field of ergonomics, supports this integration. This is achieved by demonstrating how the concepts and methods of the course of action theory, used to design new work situations when automating small hydroelectric power plants (SHPPs), leads to a broader empirical praxeology for addressing H-H (training) and H-M (automation) interfaces, or H-H-M systems. Theoretically, this article contributes to the technological pole of the Course of Action Research Programme, while in practical terms it warns Industry 4.0 professionals not to repeat the old myth (and expression) of the "unmanned factory".

\section{INDEX}

Keywords : course of action, representamen, anthropocentric design, tacit knowledge, 4.0 industry

Mots-clés : cours de l'action, representamen, projet anthropocentrique, savoir tacite, industrie 4.0

\section{AUTEURS}

\section{FRANCISCO DE PAULA ANTUNES LIMA}

Universidade Federal de Minas Gerais - UFMG ; Av. Pres. Antônio Carlos, 6627, Pampulha, 31270-901, Belo Horizonte, MG, Brasil, fpalima@ufmg.br

\section{RODRIGO RIBEIRO}

Universidade Federal de Minas Gerais - UFMG ; Av. Pres. Antônio Carlos, 6627, Pampulha, 31270-901, Belo Horizonte, MG, Brasil, rodrigoribeiro@ufmg.br

\section{MARCELLE LA GUARDIA}

Situated Consultoria e Pesquisa - Avenida Prudente de Morais, 621, Santo Antônio, 30350-143, Belo Horizonte, MG, Brasil. marcelle.laguardia.lara@gmail.com

\section{SAMIRA NAGEM}

Situated Consultoria e Pesquisa - Avenida Prudente de Morais, 621, Santo Antônio, 30350-143, Belo Horizonte, MG, Brasil. sanagemlima@gmail.com 